FTPI-MINN-14/21

UMN-TH-3346/14

\title{
Inflationary tensor fossils in large-scale structure
}

\section{Emanuela Dimastrogiovanni, ${ }^{a}$ Matteo Fasiello, ${ }^{b}$ Donghui Jeong, ${ }^{c, d}$ Marc Kamionkowski ${ }^{e}$}

${ }^{a}$ School of Physics and Astronomy, University of Minnesota, Minneapolis, MN 55455, USA

${ }^{b}$ Department of Physics, Case Western Reserve University, Cleveland, OH 44106, USA

${ }^{c}$ Department of Astronomy and Astrophysics, The Pennsylvania State University, University Park, PA 16802 USA

${ }^{d}$ Institute for Gravitation and the Cosmos, The Pennsylvania State University, University Park, PA 16802, USA

${ }^{e}$ Department of Physics and Astronomy, 3400 N. Charles St., Johns Hopkins University, Baltimore, MD 21218 USA

E-mail: ema@physics.umn.edu,mrf65@case.edu, kamion@jhu.edu,duj13@psu.edu

Abstract. Inflation models make specific predictions for a tensor-scalar-scalar three-point correlation, or bispectrum, between one gravitational-wave (tensor) mode and two densityperturbation (scalar) modes. This tensor-scalar-scalar correlation leads to a local power quadrupole, an apparent departure from statistical isotropy in our Universe, as well as characteristic four-point correlations in the current mass distribution in the Universe. So far, the predictions for these observables have been worked out only for single-clock models in which certain consistency conditions between the tensor-scalar-scalar correlation and tensor and scalar power spectra are satisfied. Here we review the requirements on inflation models for these consistency conditions to be satisfied. We then consider several examples of inflation models, such as non-attractor and solid-inflation models, in which these conditions are put to the test. In solid inflation the simplest consistency conditions are already violated whilst in the non-attractor model we find that, contrary to the standard scenario, the tensor-scalarscalar correlator probes directly relevant model-dependent information. We work out the predictions for observables in these models. For non-attractor inflation we find an apparent local quadrupolar departure from statistical isotropy in large-scale structure but that this power quadrupole decreases very rapidly at smaller scales. The consistency of the CMB quadrupole with statistical isotropy then constrains the distance scale that corresponds to the transition from the non-attractor to attractor phase of inflation to be larger than the currently observable horizon. Solid inflation predicts clustering fossils signatures in the current galaxy distribution that may be large enough to be detectable with forthcoming, and possibly even current, galaxy surveys. 


\section{Contents}

1 Introduction $\quad 1$

2 Scalar-scalar-scalar and tensor-scalar-scalar consistency conditions 2

2.1 Origin of the ccs 3

2.2 Intuitive understanding of the CCS

$\begin{array}{lll}2.3 & \text { Preview of non-attractor inflation } & 6\end{array}$

$\begin{array}{lll}3 & \text { Inflation with a Non-Attractor Phase } & 7\end{array}$

3.1 Review of linear perturbations $\quad 8$

3.2 Tensor-scalar-scalar correlator 9

4 Solid Inflation $\quad 13$

4.1 The Model 13

4.2 Perturbations 14

4.3 Violations of the consistency conditions 16

5 Observational signatures $\quad 16$

5.1 Local Power Quadrupole 17

$\begin{array}{lll}5.2 & \text { Clustering fossils } & 19\end{array}$

$\begin{array}{lll}\text { 5.2.1 Clustering fossils with the consistency relation } & 19\end{array}$

5.2.2 Clustering fossils in solid inflation 20

6 Conclusions $\quad 21$

$\begin{array}{ll}\text { A Zeroth and first order CCS } & 22\end{array}$

B The tensor-scalar-scalar action $\quad 23$

\section{Introduction}

The notion that the Universe began with a period of inflationary expansion [1-5] has been supported with a series of increasingly precise observational tests. The tests have verified that a number of characteristics of primordial density (scalar metric) perturbations -including adiabaticity, Gaussianity, and near scale-independence - agree with those in these simplest single-field slow-roll (SFSR) models. Still, inflation has even more consequences, including the prediction of a nearly scale-invariant spectrum of primordial gravitational waves [6-9]. Even though it is too early to attribute conclusively the CMB B modes $[10,11]$ detected by BICEP2 [12] to inflationary gravitational waves, evidence for a scalar spectral index $n_{s} \not \equiv 1$ $[13,14]$ provides some reason to believe that the gravitational-wave amplitude might be large. It is thus warranted to think about other obervational consequences of primordial gravitational waves and what we can learn about inflation from such observations.

In particular, there is a growing body of work on the imprints of gravitational waves on large-scale structure. Lensing by tensor modes of the galaxy distribution [15-18], CMB [1922], and 21-cm fluctuations [23-25] have been studied. However, there may also be imprints on the unlensed mass distribution. One possibility is that long-wavelength gravitational waves 
may give rise to an apparent local departure from statistical isotropy in the form of a power quadrupole [26-29] that can be observed in the CMB [30, 31] and large-scale structure [35]; some (null) CMB [32-34] and large-scale-structure [36] searches have already been carried out. There are then higher-order correlations in the density distribution, induced by coupling to gravitational waves, that can be sought [16, 24, 37-40] and also possible signatures [16, 40] in the tidally-induced intrinsic alignments of galaxies [41].

The purpose of this paper is to study the prospects to learn about inflation through the tensor-scalar-scalar (TSS) correlation. Such a correlation arises even in the simplest SFSR models [42]. This SFSR tensor-scalar-scalar bispectrum satisfies a particular consistency condition (cc) that relates the functional dependence of the TSS bispectrum on the tensor wavenumber $K$ and scalar wavenumbers $k_{1}$ and $k_{2}$ to the tensor and scalar power spectra in the squeezed limit $\left(K \ll k_{1}, k_{2}\right)$ [42-44]. This TSS consistency condition parallels an analogous consistency condition for the scalar three-point function (SSS) that is known to hold not just for SFSR inflation, but for single-clock models more generally [45-50, 52, 53]. As we show below, arguments like those for the generality of the SSS consistency condition apply also to the TSS consistency condition, and so the TSS consistency condition is generic to a fairly broad class of inflationary models.

The primordial TSS bispectrum that arises if the consistency condition is satisfied naively implies a power quadrupole that suffers a logarithmic (for a scale-independent tensor power spectrum) infrared divergence [27, 38]. However, that divergence is cancelled precisely by an analogous divergence at late times in the projection effect including lensing deflection $[28,54,55]$ leaving a finite observable power quadrupole $[28,40]$, which although nonzero turns out to be quite small. The cancellation arises, though, only if the consistency condition is satisfied. Thus, if a power quadrupole in excess of that expected from this consistency condition were to be discovered, it would rule out the vast majority of single-field inflationary models. It is therefore crucial to understand the origin, the scope, and the limits of the TSS consistency condition in inflationary setups.

Below, in Section 2, we first review the origin of the scalar-scalar-scalar cc and then how, on general grounds, the TSS consistency condition arises analogously. In Section 3 we then consider non-attractor inflation [56-58]. These models consist nominally of only a single scalar field. However, the slow-roll phase that is assumed to have been reached in SFSR models is not satisfied. The violation in these models of the slow-roll conditions implies that the scalar-field equation of motion is a second-order differential equation rather than a firstorder differential equation. The physical conditions in the Universe at some particular time are therefore not determined exclusively by the inflaton field value, and so the single-clock conditions may be violated. We calculate the TSS bispectrum and show that, intriguingly, it directly probes model-dependent information. In Section 4 we consider solid inflation [59] as another example of a model in which the consistency condition may be violated. In Section 5 we calculate the observable power quadrupole in these models and also study the prospects to discern from higher-order galaxy clustering the differences between the TSS in these models. We then conclude in Section 6.

\section{Scalar-scalar-scalar and tensor-scalar-scalar consistency conditions}

A great deal of recent attention [45-49, 52, 53] has been directed towards clarifying the origin of the consistency conditions for cosmological correlators [42]. The scope of the consistency conditions extends well beyond single-field slow-roll inflation [45]. However, as exemplified 
in Refs. [56, 57] (see also Ref. [58]), violations can occur already in single-field inflation. As we will see, these models share a specific property that causes them to evade the consistency conditions. ${ }^{1}$

The key realization is that the consistency conditions are a direct consequence of the invariance under space diffeomorphisms of the classical and quantum theory. There is more to it: crucially one need not necessarily specify the form of the action as long as the symmetry is in place and a locality requirement ${ }^{2}$ is satisfied. This latter realization is at the heart of the generalization of the results of Ref. [42] found in Ref. [45] and put on firmer ground by Ref. [49].

Whenever the locality requirement is met, the consistency conditions for a scalar (tensor) with two hard scalars at zeroth order in the soft momentum $q$ take the familiar form,

$$
\frac{\left\langle\zeta_{\vec{q}} \zeta_{\vec{p}} \zeta_{-\vec{q}-\vec{p}}\right\rangle}{P_{\zeta}(q)}=-\left(3+p_{i} \frac{\partial}{\partial p_{i}}\right) P_{\zeta}(p) ; \quad \frac{\left\langle\gamma_{\vec{q}}^{i j} \zeta_{\vec{p}} \zeta_{-\vec{q}-\vec{p}}\right\rangle}{P_{\gamma}(q)}=-\frac{1}{2} \hat{P}^{i j k l}(\hat{q}) p_{k} \frac{\partial}{\partial p_{l}} P_{\zeta}(p)
$$

where $q \ll p$ is the wavenumber of the long-wavelength mode, $p$ that of the short-wavelength modes, and $P_{\zeta}(k)$ and $P_{\gamma}(k)$, respectively, the scalar and tensor power spectra defined as

$$
\begin{array}{r}
\left\langle\zeta(\vec{k}) \zeta\left(\vec{k}^{\prime}\right)\right\rangle=(2 \pi)^{3} P_{\zeta}(k) \delta^{D}\left(\vec{k}+\vec{k}^{\prime}\right) \\
\left\langle\gamma_{i j}^{\lambda}(\vec{k}) \gamma_{i j}^{\lambda}\left(\vec{k}^{\prime}\right)\right\rangle=(2 \pi)^{3} 4 P_{\gamma}(k) \delta^{D}\left(\vec{k}+\vec{k}^{\prime}\right) .
\end{array}
$$

In words, this specific limit of a three-point function depends entirely on the behavior of the two-point correlator, regardless of the information content (i.e. interactions) one can access only at the level of the cubic action.

We give below a brief account (based mainly on [52]) of how cosmological consistency conditions such as the ones above are derived to all orders in the soft momentum $q$ as a consequence of the Slavnov-Taylor identity for spatial diffeomorphisms. For a thorough treatment, we refer the reader to Refs. [48, 52, 53].

\subsection{Origin of the ccs}

We use the fixed-time path integral of Ref. [48], a 3-D Euclidean path integral over configurations at the final time, with the wavefunction storing the "history" information. The fluctuations around an FRW background are,

$$
g_{\mu \nu}=\bar{g}_{\mu \nu}+a^{2}(t) h_{\mu \nu}=\operatorname{diag}\left(-1, a^{2}(t)\right)+a^{2}(t) h_{\mu \nu} ; \quad \phi(x, t)=\bar{\phi}(t)+\varphi(t, x) .
$$

Fixed-time correlators, to be turned into cosmological observables, can be obtained from the generating functional,

$$
Z[T, J]=\int \mathcal{D} h_{i j} \mathcal{D} \varphi|\Psi[h, \varphi, t]|^{2} \operatorname{Exp}\left(\int d^{3} x\left(h_{i j} T^{i j}+\varphi J\right)\right),
$$

where $J$ and $T$ are the currents and $\Psi$ the wave function.

\footnotetext{
${ }^{1}$ This is true at least at lowest order in the soft momentum $q$, in their simplest and model-independent formulation.

${ }^{2}$ The generic locality requirement is equivalent to adiabaticity in cosmological parlance [49].
} 
Since the theory involves general relativity plus a scalar field, both the action and the functional measure will be invariant under space diffeomorphisms (we have surrendered time-reparametrization invariance by choosing the fixed-time formalism). Requiring that the same is true for the generating functional ( $Z$ ought to be invariant under a field-redefinition) amounts to the condition [52],

$$
\begin{aligned}
0 & =\int \mathcal{D} h_{i j} \mathcal{D} \varphi|\Psi[h, \varphi, t]|^{2} e^{S_{\text {ource }}} \int \mathrm{d}^{3} x \xi^{k}\left\{(\mathrm{G} . \mathrm{F} .)_{k}-2 \partial_{j} T_{k}^{j}+\partial_{k} h_{i j} T^{i j}-2 \partial_{j}\left(h_{i k} T^{i j}\right)+\partial_{k} \varphi J\right\} \\
& =\int \mathrm{d}^{3} x \xi^{k}\left\{(\text { G.F. })_{k}-2 \partial_{j} T_{k}^{j}+\partial_{k}\left(\frac{\delta}{\delta T^{i j}}\right) T^{i j}-2 \partial_{j}\left(\frac{\delta}{\delta T^{i k}} T^{i j}\right)+\partial_{k}\left(\frac{\delta}{\delta J}\right) J\right\} Z[T, J],
\end{aligned}
$$

which is obtained by varying the gauge-fixing term and the source term within $Z$ under space diffeomorphisms,

$$
\varphi \rightarrow \varphi+\xi^{k} \partial_{k} \varphi ; \quad h_{i j} \rightarrow h_{i j}+\partial_{i} \xi_{j}+\partial_{j} \xi_{i}+\xi^{k} \partial_{k} h_{i j}+h_{j k} \partial_{i} \xi^{k}+h_{i k} \partial_{j} \xi^{k},
$$

and where $S_{\text {ource }}$ stands for the argument of the exponential in Eq. (2.5). The term (G.F.) $k$ denotes the contribution originating from the variation of the gauge-fixing term. Although essential for the general formula, this contribution plays no role in the derivation of tree-level consistency conditions for a soft tensor with two hard scalar modes that we are after.

Our starting point here has been a functional integral over the metric entries $h_{i j}$ only, while $h_{00}$ and $h_{0 i}$ have been already integrated out by solving the equation of motion. This will be essential in a few steps. Indeed, despite being handed a theory which is clearly local, integrating out $h_{00(i)}$ (alternatively known as $N_{1}, N_{i}$ in the ADM formalism and in Ref. [42]) results in spatially non-local terms (recall that, e.g., $N_{i} \sim \partial_{i} \partial^{-2}(\ldots)$ ).

From the second line in Eq. (2.6) one can see that, $\xi$ being arbitrary, the entire integrand must vanish as a result of the invariance of $Z$ under field redefinitions. It is thus convenient to introduce the effective action,

$$
\Gamma[h, \varphi]=\ln Z-\int d^{3} x\left(h_{i j} T^{i j}+\varphi J\right) .
$$

Upon choosing to work in comoving gauge, ${ }^{3}$ requiring a vanishing integral amounts to an identity of the form,

$$
\frac{1}{\alpha}\left(\vec{\nabla}^{2} \partial_{j} \gamma_{i j}+\partial_{i} \partial_{j} \partial_{k} \gamma_{j k}\right)+2 \partial_{j}\left(\frac{1}{6} \delta_{i j} \frac{\delta \Gamma}{\delta \zeta}+\frac{\delta \Gamma}{\delta \gamma_{i j}}\right)=\partial_{i} \zeta \frac{\delta \Gamma}{\delta \zeta}+\cdots,
$$

where the dots denote contributions that are higher order in $\gamma$ and thus irrelevant for the tree-level identity. The term proportional to $\alpha^{-1}$, originating from the gauge-fixing part, will not play a role in what follows.

To obtain the consistency conditions in their more familiar form, we note that the effective action $\Gamma$ is the generating function of the 1PI correlation functions. Since we are after the squeezed limit of a three-point function with a soft tensor (scalar) mode and two hard scalars, it suffices to differentiate the expression above twice with respect to $\zeta$. In Fourier space,

$$
\frac{1}{3} q_{i} \Gamma^{\zeta \zeta \zeta}(\vec{q}, \vec{p},-\vec{q}-\vec{p})+2 q^{j} \Gamma_{i j}^{\zeta \zeta \gamma}(\vec{q}, \vec{p},-\vec{q}-\vec{p})=q_{i} \Gamma_{\zeta}(p)-p_{i}\left(\Gamma_{\zeta}(|\vec{q}+\vec{p}|)-\Gamma_{\zeta}(p)\right) .
$$

\footnotetext{
${ }^{3}$ The comoving gauge is defined by $\varphi=0 ; \quad \delta_{i j}+h_{i j}=e^{2 \zeta} \hat{h}_{i j} ; \quad$ with $\operatorname{det} \hat{h}=1 ; \quad \gamma_{i j} \equiv$ $\ln \hat{h}_{i j} ; \quad \gamma_{i}^{i}=0$
} 
where $\Gamma^{\zeta \zeta \zeta}, \Gamma^{\zeta \zeta \gamma}, \Gamma_{\zeta}$ are functional derivatives of the effective action $\Gamma$ with respect to their indices (e.g., $\Gamma_{\zeta} \equiv \delta^{2} \Gamma / \delta \zeta^{2}$ ), which are proportional to, respectively, $\langle\zeta \zeta \zeta\rangle,\langle\zeta \zeta \gamma\rangle$, and $\langle\zeta \zeta\rangle$. The solution to Eq. (2.9) can be obtained [52] order by order in $q$. Once a suitable projection operator $\hat{P}$ has been found [52], the solutions for the individual vertices are,

$$
\frac{\left\langle\zeta_{\vec{q}} \zeta_{\vec{p}} \zeta_{-\vec{q}-\vec{p}}\right\rangle}{P_{\zeta}(q)}=K(\vec{p}, \vec{q})+A(\vec{p}, \vec{q}), \quad \frac{\left\langle\gamma_{\vec{q}}^{i j} \zeta_{\vec{p}} \zeta_{-\vec{q}-\vec{p}}\right\rangle}{P_{\gamma}(q)}=\frac{1}{2} \hat{P}^{i j k l}(\hat{q})\left(K_{k l}+A_{k l}\right),
$$

where $K_{i j}$ is an array completely determined out of $P_{\zeta}$ while, most importantly for us, $A_{i j}$ is an arbitrary symmetric and transverse matrix ( $K, A$ being the respective traces). The latter, in full generality, will be of the form,

$$
A_{i j}=\epsilon_{i k m} \epsilon_{j l m} q^{k} q^{l}\left(a(\vec{q}, \vec{p}) \delta^{m n}+b(\vec{q}, \vec{p}) p^{m} p^{n}\right) .
$$

Thus, exploiting one's knowledge on the symmetry of the action can prove extremely useful and deliver a relation between an $N+1$ and an $N$-point correlator. But, before a crucial hypothesis in made on $A_{i j}$, it can only take us so far. Indeed the physics that is specific to the dynamics of a given inflationary phase (let alone an inflationary model) is encoded into the functions $a, b$.

It seems reasonable to assume that $A$ starts out as a quadratic function in $q$, which would be tantamount to saying that $a, b$ are local functions. It would imply that there is an order $q^{0}$ and an order $q$ consistency condition to be derived without the need of the explicit information stored in $a, b$. If this were indeed the case, the $q^{0}$-order ccs for a soft scalar and a soft tensor would read as in Eq. (2.1), a result one can arrive at from Eq. (2.10) using the explicit solution for $K_{i j}{ }^{4}$

Crucially, as we stressed above, the theory one deals with at this point follows from integrating out the lapse and the shift function using the constraint equations. In particular, the shift is,

$$
N_{i} \sim \epsilon \frac{q_{i}}{q^{2}} \dot{\zeta}+\text { local }
$$

Now, since the action (and therefore the three-point function) is also composed of terms like the non-local contribution above, the only way to be sure that this will not result in a nonanalytic $a, b$ is to check a non-trivial property on the $\dot{\zeta}$ part, namely that its time derivative is such that this contribution becomes local; e.g., $\dot{\zeta} \sim q^{2}$.

This is precisely what happens whenever $\zeta$ is an adiabatic mode [60], and is therefore conserved outside the horizon. Conversely, as soon as $\zeta$ is not conserved, $A$ might well play a role in the consistency conditions already at order one or zero. But those orders in $q$ are exactly the ones that gave us $^{5}$ Eq. (2.1) which we now recognize as valid only for a class, albeit a very populated one, of possible cases.

\subsection{Intuitive understanding of the CCS}

We now provide a more intuitive understanding of how and why the ccs do not take the usual form, Eq. (2.1), for cases such as the one of a non-attractor solution. The familiar intuitive

\footnotetext{
${ }^{4}$ We refer the interested reader to Ref. [52] for an explicit expression. The crux of the matter here is that $K_{i j}$ is entirely determined from one's knowledge of the 2-point function only.

${ }^{5}$ The $q^{1}$ part of Eq. (2.1) has been omitted in the Introduction but can be found in Appendix A.
} 
picture is due to Maldacena [42]: the effect of e.g. a soft scalar mode on two hard scalars is that of anticipating their horizon exit by an amount $\delta t^{*}$ which is easily calculated as follows,

$$
e^{2 \zeta_{S}} e^{2 \zeta_{L}} a^{2}(t) \simeq\left(1+2 \zeta_{L}\right) e^{2 \zeta_{S}} a^{2}(t) \sim e^{2 \zeta_{S}} a^{2}\left(t+\delta t^{*}\right) \rightarrow \delta t^{*} \sim-\zeta_{L} / H,
$$

so that the overall effect on the hard modes is well approximated by $-\zeta_{L} / H \times d / d t^{*}\left(\left\langle\zeta_{S} \zeta_{S}\right\rangle\right)$, and the overall squeezed limit of the scalar three-point function is proportional to the longwavelength spectrum times the tilt of the short-wavelength spectrum. An analogous relation is found along similar lines whenever the soft mode is a tensor one.

Eq. (2.13) is a good approximation because one is expanding the exponential at a time around $t^{*}$ when the mode $\zeta_{L}$ has long left the horizon and does not depend on time anymore. If this latter condition were not satisfied, one would have to keep track of the history of $\zeta_{L}$ since it left the horizon.

Ref. [45] present a case for consistency conditions in single-clock solutions whose background is a dynamical attractor. In non-attractor inflation, the evolution of the inflaton has not yet reached the attractor phase - the decaying mode has not yet decayed away. The physical conditions at some point in the Universe therefore cannot, as in single-clock inflation, be specified entirely by a single parameter.

The perspective of Ref. [45] is to reabsorb $\zeta_{L}$ into the comoving space variable -i.e., in the metric $e^{2 \zeta_{L}} d \mathbf{x}^{2}=d \tilde{\mathbf{x}}^{2}$ - so that one can then effectively trade $d / d \zeta_{L}$ with $x \cdot d / d x$ in calculating the effect of a soft modes on two hard ones. ${ }^{6}$ This trading evidently becomes off limits as soon as $\zeta_{L}$ is time dependent thus taking us back to the locality (or adiabaticity) argument discussed above.

\subsection{Preview of non-attractor inflation}

Before detailing its dynamics, we give a qualitative picture of the non-attractor inflationary phase from the perspective of ccs violation. As shown in Ref. [57], the observable quantity $\langle\zeta \zeta \zeta\rangle$ violates the ccs already at order $q^{0}$. As is clear from Eq. (2.11) then, this can only ${ }^{7}$ result from non-locality either in the function $a(q, p)$ or in the function $b(q, p)$.

What we are after here is instead the consistency relation (or violation thereof) of the observable $\langle\gamma \zeta \zeta\rangle$. As a result, the role of the projection operator $\hat{P}^{i j k l}$ in Eq. (2.10) is of the utmost importance. In might indeed happen that $\hat{P}$ projects out the non-analyticity of $a$ or $b$ from contributing to the tensor-scalar-scalar three-point function at some (or all) orders in $q$. As it turns out, this is indeed what happens for the non-attractor model under scrutiny in this manuscript and therefore, in this strict sense, one should say that the TSS three-point function does not violate the consistency conditions. Nevertheless, as we shall see, one is able to probe the model-dependent part of the inflationary mechanism encoded in $A_{i j}$ by accounting for the contribution of interaction terms which are simply slow-roll suppressed in the typical attractor models.

The key point is that, in the non-attractor phase, the small parameter $\epsilon$ is strongly time dependent, and it is precisely this time dependence that can compensate for the slowroll suppression. The three-point-function contributions of these interactions scale like $q^{2}$ and are therefore ideal candidates to probe the content of $A_{i j}$, be it the non-local or the analytic/adiabatic one. We will see below how there are clear-cut ways to discern whether these $q^{2}$ terms contribute to $A_{i j}$, or instead to the model-independent piece $K_{i j}$.

\footnotetext{
${ }^{6}$ The difference, as well as the overall analogy with Ref. [42], where $\zeta_{L}$ is embedded into the scale factor, is clear.

${ }^{7} K_{i j}$ is always a local function.
} 


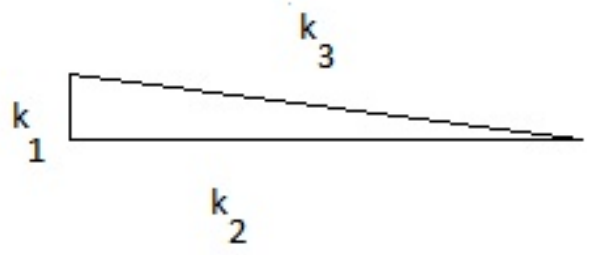

Figure 1. Squeezed configuration for the momenta: $k_{1} \ll k_{2} \simeq k_{3}$.

In all that follows, the characterization of the TSS signal of the non-attractor model as violating the ccs is to be understood only in the sense above, that is: the squeezed limit is probing model-dependent information of the non-attractor model through contributions to the three-point function which are not naively slow-roll suppressed. The TSS carries an imprint of the non-attractor phase that cannot be extracted from the power spectrum nor from its derivatives. This is in contradistinction to the vast majority of inflationary models, including SFSR.

\section{Inflation with a Non-Attractor Phase}

As discussed above, non-attractor models are those where the decaying solution for the inflaton equation of motion has not yet fully decayed. Following previous work on nonattractor inflation, we consider k-inflation [61] models described by a Lagrangian density $P(X, \phi)$ that is a function of the inflaton $\phi$ and $X \equiv-1 / 2\left(\partial_{\mu} \phi\right)^{2}$. The model is parametrized by the quantities,

$$
c_{s}^{2} \equiv \frac{P_{, X}}{P_{, X}+2 X P_{, X X}}, \quad \epsilon \equiv-\frac{\dot{H}}{H^{2}}=\frac{X P_{, X}}{M_{P}^{2} H^{2}}, \quad \eta \equiv \frac{\dot{\epsilon}}{H \epsilon}=\frac{\ddot{\phi}}{H \dot{\phi}}\left(1+\frac{1}{c_{s}^{2}}\right)+\frac{\dot{\phi} P_{, X \phi}}{H P_{, X}}+2 \epsilon .
$$

The sound speed $c_{s}$ varies between 0 and 1 . The slow-roll parameters $\epsilon$ and $\eta$ are generally small and vary slowly with time, although these assumptions are modified during the nonattractor phase.

Ref. [57] presents a model of non-attractor inflation that involves a Lagrangian density,

$$
P(X, \phi)=X+\frac{X^{\alpha}}{M^{4(\alpha-1)}}-V(\phi), \quad V(\phi)=V_{0}+v\left(\frac{\phi}{M_{P}}\right)^{\beta},
$$

where $\alpha, V_{0}, v$, and $\beta$ are initially free parameters and $M_{P} \equiv 1 / \sqrt{8 \pi G_{N}}$ is the reduced Planck mass. During the first phase of inflation, the system evolves around a non-attractor background: the inflaton rolls up its potential, progressively slowing down (part (a) of Fig(2)). This first phase can be followed by an attractor inflationary phase with the inflaton field rolling back down its potential on the same $\phi>0$ side ("undershoot case", see part (b) of Fig. 2) or alternatively by it going over the top of the potential and rolling down on the opposite side ("overshoot case", part (c) of Fig. 2).

For inflation to occur, the constant contribution $V_{0}$ to the potential must dominate the total energy density during inflation; i.e., $3 H^{2} M_{P}^{2} \simeq V_{0}$. The kinetic energy of the inflaton 

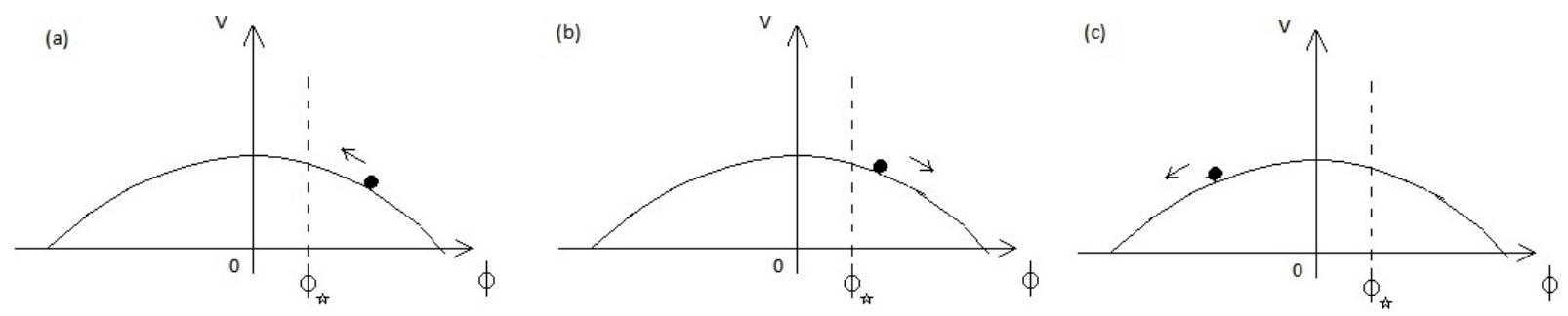

Figure 2. A qualitative representation in the potential-scalar field plane of the non-attractor phase (a), and of the attractor phase, in the form of the (b) undershoot and overshoot (c) cases. The scalar field begins with a positive value. In the undershoot case, the field reaches a value $\phi_{*}>0$ at the point where its velocity becomes null, so it rolls down on the same side of the potential (b). In the overshoot case, the system has enough kinetic energy to go over the top of the potential and roll down the other side $(c)$.

must initially be large enough to induce the field to roll up; if one considers values for the exponent $\alpha \gg 1$ in Eq. (3.2), then large values of $|\dot{\phi}|$ imply that the kinetic Lagrangian is initially dominated by the non-canonical term, $X^{\alpha} / M^{4(\alpha-1)}$. Having a large value for $\alpha$, together with $X^{\alpha} / M^{4(\alpha-1)} \gg X$, results in a sound speed, $c_{s}^{2} \simeq(2 \alpha-1)^{-1} \lesssim 1$. Following Ref. [57], we choose $\alpha>1$. To simplify, we assume that both $\eta$ and $c_{s}$ are approximately constant during inflation. If $\eta$ is indeed a constant, the other slow-roll parameter $\epsilon$ would go like $\epsilon \sim a^{\eta}$ so that an $\eta \ll 1$ would result in an approximately time-independent $\epsilon$ during inflation. However, as we will see from the analysis of linear perturbations, near scale invariance of the power spectrum in this model requires a large $\eta \simeq-6$, and hence a nonnegligible time dependence for $\epsilon$. Ref. [57] considered the ansatz $\phi \sim a^{\kappa}$ for the homogeneous evolution of the inflaton, where $\kappa$ is a constant. One then finds,

$$
\beta=2 \alpha=1+\frac{1}{c_{s}^{2}}, \quad v=-\frac{M^{4}}{c_{s}^{2}}\left(\frac{V_{0} \kappa^{2}}{6 M^{4}}\right)^{\alpha}\left(1+\frac{3 c_{s}^{2}}{\kappa}\right), \quad \epsilon \sim a^{2 \alpha \kappa} \rightarrow \kappa \simeq \frac{\eta}{2 \alpha} .
$$

For given values of $\eta$ and $c_{s}$, we are left with two independent parameters; e.g., $V_{0}$ and $v$. As anticipated, $\eta$ is fixed by scale invariance while the value of $c_{s}$ is essential to determine the amplitude of non-Gaussianity. Notice that $v<0$, so the potential $V(\phi)$ has a concave shape as in Fig. (2). Also $\kappa<0$ and, as a consequence, both $|\phi|$ and $|\dot{\phi}|$ are decreasing functions of time during this initial phase. The ansatz $\phi \sim a^{\kappa}$ and the constraints on the parameters derived from it represent an analytic solution that complies with the dynamics of a non-attractor phase, but they are not a necessary condition for a non-attractor phase.

In the undershoot situation, the field stops before reaching zero value. Therefore, as we approach the turnaround point $\left(\phi_{*}\right)$, the $X^{\alpha}$ contribution to the kinetic energy becomes progressively negligible compared to $X$. The condition that defines the end of the nonattractor phase is $\left(\phi_{*} / M_{P}\right) \simeq(\sqrt{6} /|\kappa|)\left(M^{2} / \sqrt{V_{0}}\right)$. For $t>t_{*}$ the system transitions to slowroll inflation during which the curvature perturbation is conserved on superhorizon scales.

\subsection{Review of linear perturbations}

We decompose the metric fluctuations using the ADM formalism [62],

$$
d s^{2}=-N^{2} d t^{2}+h_{i j}\left(d x^{i}+N^{i} d t\right)\left(d x^{j}+N^{j} d t\right) .
$$


We work in comoving gauge, setting to zero the fluctuations of the inflation field $(\delta \phi=0)$. In this gauge, the spatial part of the metric tensor has the form, $h_{i j}=a^{2} e^{2 \zeta}\left(e^{2 \gamma}\right)_{i j}$, where $a(t)$ is the scale factor, $\zeta(t, \vec{x})$ is the scalar fluctuation, and $\gamma_{i j}(\vec{x}, t)$ is the transverse traceless tensor perturbation (with $\partial_{i} \gamma_{i j}=\gamma_{i i}=0$ ).

Following the usual treatment, the mode function for the Fourier component $\zeta_{k}(\tau)$ during the attractor phase is

$$
\zeta_{k}=\frac{v_{k}}{z}=\frac{H_{*}}{M_{P}} \frac{e^{-i k c_{s} \tau}}{\sqrt{4 \epsilon_{*} c_{s} k^{3}}}\left(1+i k c_{s} \tau\right)
$$

In the non-attractor case, the time variation of $\epsilon$ (recall $\epsilon \sim a^{-6}$ ) leads to a different mode function [57],

$$
\zeta_{k}(\tau)=C_{k} \sqrt{\frac{2}{\pi}} \frac{e^{-i k c_{s} \tau}}{\left(-k c_{s} \tau\right)^{3}}\left(-1-i k c_{s} \tau\right), \quad\left|C_{k}\right|^{2} \equiv \frac{H_{*}^{2}}{M_{P}^{2}}\left(\frac{\pi}{8}\right) \frac{k^{3} c_{s}^{5} \tau_{*}^{6}}{\epsilon_{*}},
$$

where a "*" indicates quantities computed at the time $\tau_{*}$ when the non-attractor phase ends. Unlike the attractor case, on super-horizon scales $\zeta$ is not conserved: $\dot{\zeta}=3 H \zeta$. From Eq. (3.6) one can compute the power spectrum at the end of the non-attractor $\left(\tau=\tau_{*}\right)$ era for modes that are already super-horizon by that time $\left(\left|k c_{s} \tau_{*}\right| \ll 1\right)$. It is $P_{\zeta}(k)=$ $\left(4 k^{3}\right)^{-1}\left(H_{*} / M_{P}\right)^{2}\left(\epsilon_{*} c_{s}\right)^{-1}$. The tensor power spectrum is likewise $P_{\gamma}(k)=\left(1 / k^{3}\right)\left(H_{*} / M_{P}\right)^{2}$.

\subsection{Tensor-scalar-scalar correlator}

Prior work considered the scalar-scalar-scalar three-point function in $k$-inflation $[57,83-86]$ as well as the scalar-tensor-tensor correlator [87]. Here we calculate the squeezed limit of the primordial tensor-scalar-scalar bispectrum in $k$-inflation and in particular in the nonattractor model $[57,86]$, focusing on modes that left the horizon before the beginning of the attractor phase ${ }^{8}$.

The Schwinger-Keldysh (or in-in) formula [63] (see also Ref. [64] for a detailed and comprehensive review of the formalism) is the standard tool to calculate cosmological correlation functions. In particular, the tree-level contribution to the diagram depicted in Fig. 3 is,

$$
\left\langle\gamma\left(\tau_{0}\right) \zeta\left(\tau_{0}\right) \zeta\left(\tau_{0}\right)\right\rangle=-i \int_{-\infty}^{\tau_{0}} d \tau\left\langle\left[\gamma\left(\tau_{0}\right) \zeta\left(\tau_{0}\right) \zeta\left(\tau_{0}\right), H_{\gamma \zeta^{2}}(\tau)\right]\right\rangle
$$

where [,] denotes a quantum commutator and $H_{\gamma \zeta^{2}}=-L_{\gamma \zeta^{2}}$ is the Hamiltonian density (which can be obtained from Appendix B) to third order in fluctuations and $\tau_{0}$ is the time of observation, so $\tau_{0}>\tau_{*}$. The time integral in Eq. (3.7) can be split into two integrals, respectively for $\tau \in\left(-\infty, \tau_{*}\right)$ and $\tau \in\left(\tau_{*}, \tau_{0}\right)$. For the first integral we use the non-attractor solution, Eq. (3.6), and take $\epsilon \sim a^{\eta}$; for the second integral the attractor solution, Eq. (3.5) is employed, and $\epsilon$ is treated as a constant and evaluated at $\tau_{*}$.

The Fourier-space field operators for the scalar and tensor perturbations are

$$
\zeta_{\vec{k}}(t) \equiv a_{\vec{k}} \zeta_{k}(t)+a_{-\vec{k}}^{\dagger} \zeta_{k}^{*}(t), \quad \gamma_{i j, \vec{k}}(t) \equiv \sum_{\lambda= \pm} \epsilon_{i j}^{\lambda}(\hat{k})\left[b_{\vec{k}}^{\lambda} \gamma_{k}(t)+\left(b^{\lambda}\right)_{-\vec{k}}^{\dagger} \gamma_{k}^{*}(t)\right]
$$

\footnotetext{
${ }^{8}$ The tensor-scalar-scalar correlator has also been calculated in unpublished work by A. H. Tajdini and H. Firouzjahi.
} 


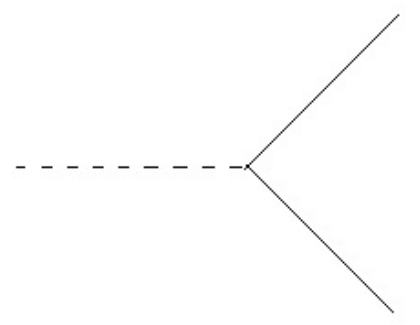

Figure 3. Diagrammatic representation of the contribution in Eq. (3.13) to the tensor-scalar-scalar correlator. The dashed line is the graviton propagator, continuous lines are the scalars.

and the creation/annihilation operators for the scalar and the tensor sectors obey the usual commutation relations. The scalar wavefunction was given in Eq. (3.6), and the tensor wavefunction is the standard one,

$$
\gamma_{k}(\tau)=\frac{H_{*}}{M_{P} \sqrt{k^{3}}}(1+i k \tau) e^{-i k \tau}
$$

where $d \tau=a d t$ is the conformal time and " * " as usual indicates quantities evaluated at the end of the non-attractor phase.

We now evaluate Eq. (3.7) to obtain the tensor-scalar-scalar bispectrum. Following Ref. [52], we define a primed three-point correlator $\langle\cdots\rangle^{\prime}$ by

$$
\left\langle\gamma_{\vec{k}_{1}}^{\lambda} \zeta_{\vec{k}_{1}} \zeta_{\vec{k}_{2}}\right\rangle \equiv(2 \pi)^{3} \delta_{D}^{(3)}\left(\vec{k}_{1}+\vec{k}_{2}+\vec{k}_{3}\right)\left\langle\gamma_{\vec{k}_{1}}^{\lambda} \zeta_{\vec{k}_{1}} \zeta_{\vec{k}_{2}}\right\rangle^{\prime}
$$

We then define the tensor-scalar-scalar bispectrum $\mathcal{B}\left(k_{1}, k_{2}, k_{3}\right)$ by

$$
\left\langle\gamma_{\vec{k}_{1}}^{\lambda} \zeta_{\vec{k}_{2}} \zeta_{\vec{k}_{3}}\right\rangle^{\prime} \equiv \epsilon_{i j}^{\lambda}\left(\hat{k}_{1}\right) \hat{k}_{2 i} \hat{k}_{3 j} \mathcal{B}\left(k_{1}, k_{2}, k_{3}\right)
$$

where $\epsilon_{i j}^{\lambda}\left(\hat{k}_{1}\right)$ is the polarization tensor of the tensor mode. Connection with the functions $K_{i j}$ and $A_{i j}$ in Section 2 can be made by identifying,

$$
k_{2 i} k_{3 j} \mathcal{B}\left(k_{1}, k_{2}, k_{3}\right)=\frac{1}{2} P_{\gamma}\left(k_{1}\right)\left(K_{i j}+A_{i j}\right) .
$$

We first evaluate Eq. (3.7), with the interactions given in the first line of Eq. (B.2). We label this correlator with a subscript "int" to distinguish it from the contributions to the total tensor-scalar-scalar correlator arising from the field redefinitions (we further elaborate on these terms later in this Section and report them in Appendix B),

$$
\mathcal{B}\left(k_{1}, k_{2}, k_{3}\right)_{\text {int }}=\mathcal{B}_{[\gamma \partial \zeta \partial \zeta]}\left(k_{1}, k_{2}, k_{3}\right)+\mathcal{B}_{\left[\partial^{2} \gamma \partial \chi \partial \chi\right]}\left(k_{1}, k_{2}, k_{3}\right)+\mathcal{B}_{[\dot{\gamma} \partial \zeta \partial \chi]}\left(k_{1}, k_{2}, k_{3}\right)
$$

The first interaction, $(\gamma \partial \zeta \partial \zeta)$, is the standard attractor-phase result. This can be easily anticipated by noticing that the integrand function for the non-attractor phase part of the integral is equal to the integrand function of the attractor period; indeed for the non-attractor phase the non-standard time dependence of the wave functions for the scalar 
fluctuations and the novel time dependence of the slow-roll parameter cancel out to leave the typical expressions that apply to the usual phase,

$$
\epsilon \times\left(\zeta^{\text {non-attr }}\right)^{2}=\epsilon_{*} \frac{\tau^{6}}{\tau_{*}^{6}} \times\left(\zeta^{a t t r} \frac{\tau_{*}^{3}}{\tau^{3}}\right)^{2}=\epsilon_{*} \times\left(\zeta^{a t t r}\right)^{2} .
$$

The result for this first contribution in the squeezed limit, $k_{1} \ll k_{2} \sim k_{3}$, is

$$
\mathcal{B}_{[\gamma \partial \zeta \partial \zeta]}\left(k_{L}, k_{S}, k_{S}\right)=\frac{3}{8} \frac{H_{*}^{4}}{M_{P}^{4}}\left(\frac{1}{\epsilon_{*} c_{s}}\right)\left(\frac{1}{k_{S}^{3} k_{L}^{3}}\right)
$$

where we redefined the momenta as $k_{1}=k_{L}$ (long-wavelength mode) and $k_{2}=k_{S}$ (shortwavelength mode). The result in Eq. (3.15) satisfies the consistency relation for tensor-scalarscalar correlators to order $\mathcal{O}\left(k_{L}^{0}\right)$,

$$
\mathcal{B}_{\text {c.r. }}\left(k_{L}, k_{S}, k_{S}\right) \equiv-\frac{1}{2} P_{\gamma}\left(k_{L}\right) P_{\zeta}\left(k_{S}\right) \frac{\partial \ln P_{\zeta}\left(k_{S}\right)}{\partial \ln k_{S}} .
$$

The remaining two interactions amount to,

$\mathcal{B}_{\left[\partial^{2} \gamma \partial \chi \partial \chi\right]}\left(k_{L}, k_{S}, k_{S}\right)+\mathcal{B}_{[\dot{\gamma} \partial \zeta \partial \chi}\left(k_{L}, k_{S}, k_{S}\right)=\frac{297}{32} \frac{H_{*}^{4}}{M_{P}^{4}}\left(\frac{1}{\epsilon_{*} c_{s}}\right)\left(\frac{1}{k_{L}^{3} k_{S}^{3}}\right) \frac{\epsilon_{*}}{\left(\tau_{*} c_{s} k_{S}\right)^{6}}\left(\frac{k_{L}}{c_{s} k_{S}}\right)^{2}$.

Although this contribution is suppressed relative to that in Eq. (3.15) by a power of $\epsilon_{*}$ and by a coefficient $\left(k_{L} / c_{s} k_{S}\right)^{2}$, it is also characterized by a factor $\left(k_{S} c_{s} \tau_{*}\right)^{-6}$. The latter, for modes that left the horizon ${ }^{9}$ before $\tau_{*}$, provides an enhancement.

This term then has the potential to provide an important contribution to the squeezed limit of the observable $\langle\gamma \zeta \zeta\rangle$. It is quadratic in the soft momentum $k_{L}$ and it therefore escapes the familiar consistency condition as written in Eq. (2.1).

In fact, already in the standard attractor-phase scenario, one would typically have terms which scale just like Eq. (3.17), that is as $\left(k_{L} / k_{S}\right)^{2}$. The difference now relies on the fact that there is a $\tau^{*}$ dependence: it is the imprint of a past non-attractor phase. It shows how the novel time dependence characterizing the wavefunction and the slow-roll parameters during the initial inflationary stage can counteract the slow-roll suppression.

Eq. (3.17) does not exhaust all the contributions at second order in slow roll. The complete calculation requires the next-to-leading order slow-roll corrections of the first interaction term in Eq. (B.2). However, just as in the standard scenario, Eq. (3.17) represents a typical contribution to the three-point correlator, and it is as such that we calculate its contribution to the observables in Section 5. As for field redefinitions like those in Eqs. (B.4) and (B.5), they do certainly contribute but they also fall into the "late time" argument given above. They therefore do not provide a source to directly probe the $\tau_{*}$ dependence of the three-point function.

The tensor-scalar-scalar bispectrum has contributions also from the field redefinitions Eqs. (B.4)-(B.5). However, their contributions are sub-leading compared to Eq. (3.13). For the sake of completeness, we report their expressions in Appendix B.

Before continuing we note that the interaction terms $\dot{\gamma} \partial \zeta \partial \chi, \partial^{2} \gamma \partial \chi \partial \chi$, precisely those probing the model-dependent part of the squeezed limit, would be, in the attractor phase,

\footnotetext{
${ }^{9}$ Remember, throughout this work, we are after modes $k_{S}$ and $k_{L}$ that are super-horizon by the time the non-attractor era ends.
} 
clearly suppressed. It is the time dependence of $\epsilon$ in the non-attractor case that is able to counteract the suppression. However, this counteraction may not go on forever, in the sense that it cannot be that increasingly $\epsilon$-suppressed terms that keep playing an important role in the result. These considerations provide a bound on the value of $k_{s} \tau_{*}$ simply springing from the consistency of the slow-roll expansion.

We now comment on the slow-roll expansion and on the magnitude of the contributions in Eqs. (3.15) and (3.17). In slow-roll, given each interaction, its contributions to higher-order correlators can be expanded in powers of the small perturbative parameter $\epsilon$. As a simple consistency criterion on this expansion, one requires that contributions given by increasing orders in $\epsilon$ get smaller and that the expansion converges. This is easily implemented during the attractor phase, when $\epsilon$ is constant. The time dependence of $\epsilon$ characterizing the nonattractor stage ought not to spoil this feature: higher-order slow-roll corrections cannot become leading.

There are three expansion parameters to keep in mind: $\epsilon, k_{L} /\left(c_{s} k_{S}\right)$ (we are probing the squeezed limit), and $k_{S} c_{s} \tau_{*}$, which is smaller than unity for modes that exited during the non-attractor phase. The result in Eq. (3.15), for instance, represents the leading-order contribution in $k_{L} /\left(c_{s} k_{S}\right)$, from the interaction $\gamma \partial \zeta \partial \zeta$. Corrections to Eq. (3.15) from this interaction include higher powers of $k_{L} /\left(c_{s} k_{S}\right)$,

$$
\mathcal{B}_{[\gamma \partial \zeta \partial \zeta]} \sim \frac{1}{k_{S}^{3} k_{L}^{3}}\left[1+\mathcal{O}\left(\frac{k_{L}}{c_{s} k_{S}}\right)^{n}\right], \quad(n>0),
$$

which one neglects in the squeezed limit. Similarly, Eq. (3.17) represents the leading-order contribution in powers of $k_{L} /\left(c_{s} k_{S}\right)$ for the other two interactions, $\partial^{2} \gamma \partial \chi \partial \chi$ and $\dot{\gamma} \partial \zeta \partial \chi$. Note that Eq. (3.17) is also the leading term in powers of $k_{S} c_{s} \tau_{*}$,

$$
\mathcal{B}_{\left[\partial^{2} \gamma \partial \chi \partial \chi\right]+[\dot{\gamma} \partial \zeta \partial \chi]} \sim \frac{\epsilon_{*}}{k_{S}^{3} k_{L}^{3}}\left[\left(\frac{k_{L}}{c_{s} k_{S}}\right)^{2}+\mathcal{O}\left(\frac{k_{L}}{c_{s} k_{S}}\right)^{2+n}\right]\left[\frac{1}{q^{6}}+\mathcal{O}\left(q^{m}\right)\right], \quad(n>0, m \geq 0),
$$

where we defined $q \equiv\left(-k_{S} c_{s} \tau_{*}\right)$. To enforce the criterion on typical-interaction terms at each order in slow-roll we proceed as follows: we require the $\gamma \partial \zeta \partial \zeta$ contribution to be larger than its counterpart at higher powers of $\epsilon$. We do the same for $\partial^{2} \gamma \partial \chi \partial \chi, \dot{\gamma} \partial \zeta \partial \chi$. Also, since the term in Eq. (3.17) is $\epsilon_{*}$ suppressed with respect to the one in Eq. (3.15), we require the latter be larger than the former.

All these conditions are readily met by requiring,

$$
q^{6}>\epsilon_{*},
$$

which is a reflection of the fact that a longer duration for the non-attractor phase will inevitably lead to a more marked imprint on the subsequent eras. Eq. (3.20) leads then to a (conservative) bound on the duration of such a stage. Notice that this condition arises naturally also from some simple considerations. One denotes by $N_{*}$ the total number of $e$ foldings, $e^{N_{*}}=\left(a_{*} / a_{i n}\right)$, for the non-attractor phase, where $a_{*}=a\left(\tau_{*}\right)\left(\tau_{*}\right.$ being the time at which the non-attractor phase ends) and $a_{i n}$ is the value of the scale factor at the beginning of the non-attractor phase. Considering modes that exit the horizon before the end of the non-attractor phase, one writes

$$
-k_{S} c_{s} \tau_{*}=\frac{a_{S} H}{a_{*} H}=\frac{a_{S}}{a_{*}}>\frac{a_{i n}}{a_{*}}=e^{-N_{*}},
$$


where $a_{S}$ is the value of the scale factor at the time the mode $k_{S}$ leaves the horizon, and $c_{s} k_{S}=a_{S} H$. During the non-attractor phase the parameter $\epsilon \equiv-\dot{H} / H^{2}$ is a decreasing function of time, $\epsilon(\tau)=\epsilon_{*}\left(\tau / \tau_{*}\right)^{6}$, for $\tau<\tau_{*}$. Requiring that $\epsilon$ is a small quantity for the whole duration of the non-attractor phase (and of course later as well), then $\epsilon_{*}<e^{-6 N_{*}}$, which is consistent with Eqs. (3.21) and (3.20).

In and of itself, the previous equation does not provide a strong constraint on the parameter space of the model under scrutiny. However, if we take a tensor-to-scalar ration $r=16 \epsilon_{*} c_{s} \sim 0.1$ and assume $c_{s}=1$, then we find a non-attractor phase that lasts about one $e$-fold. A longer non-standard stage requires a smaller $\epsilon_{*}$.

To get a grasp of how the relative size of the various interaction contributions translate into bounds one may, for example, require that the contribution in Eq. (3.17) be smaller but within the same order of magnitude of Eq. (3.15). This would result in a ratio $k_{L} / k_{S}$ which is still small, of order $1 / 10$.

\section{Solid Inflation}

\subsection{The Model}

Recently, an intriguing inflationary model has been put forward in Ref. [59]. Although the symmetry-breaking pattern of this theory is far from that in the standard picture, it nevertheless results in a well-controlled inflationary phase that produces signatures quite distinct from those of the standard scenario. Of interest here is the fact that this model entails no adiabatic modes during the entirety of the inflationary era. We begin by reprising the theory and then move on to discuss a consistency-condition-violating scalar-scalar-tensor three-point function. For a more thorough treatment we refer the reader to the original work in Ref. [59] and follow-ups in Refs. [65-67] (for more on SI see also [68, 69]).

Solid inflation describes an homogeneous and isotropic cosmological background even though it involves a scalar-field background that is space-dependent and breaks spatialtranslation and rotational invariance. The key point is to require some "internal" symmetries in field space so as to make the combined spatial+internal transformations a symmetry of the theory.

The symmetry-breaking background is $\left\langle\phi^{I}\right\rangle=x^{I}$, but the theory is also endowed with internal symmetries under,

$$
\phi^{I} \rightarrow \phi^{I}+A^{I} ; \quad \phi^{I} \rightarrow O_{J}^{I} \phi^{J}, \quad\left(O_{J}^{I} \in S O(3)\right) .
$$

One can think of the $\phi^{I}$ 's as internal comoving coordinates around which, once time dependence is accounted for, describe a solid and its volume-element position, $\bar{x}=\bar{x}\left(t, \phi^{I}\right)$. But at each $t$ this might be inverted to give $\phi^{I}=\phi^{I}(t, \bar{x})$. The latter form turns out to be more convenient, as one handles spacetime symmetries in the more familiar fashion. The treatment then reduces, in flat space, ${ }^{10}$ to that of a relativistic low-energy effective theory of the three Poincare scalars $\phi^{I}$ endowed with a number of symmetries (those dictated by the request of homogeneity and isotropy in the background).

These simple considerations coupled with Eq. (4.1) are enough to greatly constrain the form of the action at lowest order in the derivative expansion,

$$
S=\int d^{4} x F(X, Y, Z) \equiv \int d^{4} x F\left([B], \frac{\left[B^{2}\right]}{[B]^{2}}, \frac{\left[B^{3}\right]}{[B]^{3}}\right)
$$

\footnotetext{
${ }^{10}$ The extension to cosmological solutions with dynamical gravity is not particularly involved, but we omit details here.
} 
where $B^{I J}=\left(\partial_{\mu} \phi^{I}\right)\left(\partial^{\mu} \phi^{J}\right)$ and the first invariant (under Eq. (4.1)) variable, $X \equiv[B] \equiv$ $\operatorname{Tr}\left[B^{I J}\right]$, has been chosen to keep track of the overall size of the system. The background will spontaneously break some of the symmetries, a breaking to which Goldstone bosons will be associated. ${ }^{11}$ These are fluctuations $\phi^{I}=\phi^{I}\left(x^{I}+\pi^{I}\right)$ around the background solution $\phi^{I}\left(x^{I}\right)$.

\subsection{Perturbations}

Expanding in the flat-space action one gets, at quadratic order [59],

$S^{(2)}=\int d^{4} x\left[-\frac{1}{3} F_{X} X \dot{\vec{\pi}}^{2}+\left(\frac{1}{3} F_{X} X+\frac{6}{27}\left(F_{Y}+F_{Z}\right)\right)\left(\partial_{i} \pi_{j}\right)^{2}+\left(\frac{1}{9} F_{X X} X^{2}+\frac{2}{27}\left(F_{Y}+F_{Z}\right)\right)(\vec{\nabla} \cdot \vec{\pi})^{2}\right]$,

where the derivatives $F_{(.)}$are calculated on the background. These Goldstone modes are phonons of the solid, and they can be split into longitudinal and transverse components $\bar{\pi}_{L}$ and $\bar{\pi}_{T}$ with associated speeds of propagation [59],

$$
c_{L}^{2}=1+\frac{2}{3} \frac{F_{X X} X^{2}}{F_{X} X}+\frac{8}{9} \frac{\left(F_{Y}+F_{Z}\right)}{F_{X} X}, \quad c_{T}^{2}=1+\frac{2}{3} \frac{\left(F_{Y}+F_{Z}\right)}{F_{X} X} .
$$

Gravity can be turned on by promoting $\eta_{\mu \nu}$ to a more general $g_{\mu \nu}$, introducing the corresponding measure and minimally coupling gravity to the $\phi^{I}$ fields. The resulting stress-energy tensor is indeed homogeneous and isotropic as desired. To sustain a superluminality-free slow-roll phase without strong-coupling issues, the parameters must satisfy [59],

$$
\epsilon=\frac{3}{a^{2}} \frac{F_{X}}{F}=\frac{\partial \log F}{\partial \log X} \ll 1 \quad \text { (s.r.); } \quad 0<F_{Y}+F_{Z}<\frac{3}{8} \epsilon|F| \quad \text { (luminality). }
$$

The smallness of $F_{X}$ can be linked with the breaking of a scaling symmetry (under which both $Y, Z$ are already invariant, as one might expect, $X$ being the only variable that carries the overall size information of the system) and therefore be stable under quantum corrections. This is not true, though, for the latter condition in Eq. (4.4) which is at this stage an assumption that might require some tuning. The validity of perturbation theory requires that the condition,

$$
\epsilon c_{L}^{3} \gg\left(H / M_{P}\right)^{2 / 3} \quad \text { (pert.theory), }
$$

holds. The smallness of the slow-roll parameter $s=\dot{c}_{s} /\left(H c_{s}\right)$ is automatic if the above conditions are met and therefore generates no more bounds.

One of them is the fact that the "clock" regulating a beginning and signaling the end for inflation is entirely due to the metric field. One might choose a number of gauge-invariant observables as the physical clock, such as the energy density and pressure, or $X$ itself, but the unperturbed $\phi^{I} \mathrm{~s}$ by themselves have no time dependence. This is in stark contrast with the physical-clock role played by the inflaton in most inflationary mechanisms. It is a fact of consequence because some of the predictions of the model will depend on which variable is used to signal the end of inflation and trigger reheating.

The Goldstone-boson picture has been already employed in the literature to describe inflation, most notably in the effective-field-theory approach to inflation [70]. In this approach,

\footnotetext{
${ }^{11}$ The logic here is the same as in the effective-field-theory approach but the symmetry-breaking pattern is different and this has crucial consequences, some of which we shall report below.
} 
the symmetry-breaking pattern is the more familiar one where it is time diffeomorphisms that are broken by the Goldstone; one can show ${ }^{12}$ that the two scenarios cannot be mapped into each other.

In solid inflation tensor and scalar gauge-invariant fluctuations are not adiabatic and are not conserved outside the horizon. This is, as we have seen, the scenario where violations of consistency conditions take place. The physical picture as to why this happens is [59] that adiabatic mode can be reabsorbed by a time shift of the background if the wavelength of the fluctuation is large enough. However, solid can experience anisotropic stresses, non-scalar perturbations, that cannot be re-absorbed as a time shift.

With these considerations it is not surprising that the solutions to the equations of motion that emerge from the following quadratic scalar, vector and tensor Lagrangians are not constant outside the horizon:

$$
\begin{aligned}
S^{(2)} & =S_{\gamma}^{(2)}+S_{T}^{(2)}+S_{L}^{(2)} \\
S_{\gamma}^{(2)} & =\frac{1}{4} M_{P}^{2} \int d t d^{3} x a^{3}\left[\frac{1}{2} \dot{\gamma}_{i j}^{2}-\frac{1}{2 a^{2}}\left(\partial_{m} \gamma_{i j}\right)^{2}+2 \dot{H} c_{T}^{2} \gamma_{i j}^{2}\right] \\
S_{T}^{(2)} & =M_{P}^{2} \int d t \int_{\vec{k}} a^{3}\left[\frac{k^{2} / 4}{1-k^{2} / 4 a^{2} \dot{H}}\left|\dot{\pi}_{T}^{i}\right|^{2}+\dot{H} c_{T}^{2} k^{2}\left|\pi_{T}^{i}\right|^{2}\right] \\
S_{L}^{(2)} & =M_{P}^{2} \int d t \int_{\vec{k}} a^{3}\left[\frac{k^{2} / 3}{1-k^{2} / 3 a^{2} \dot{H}}\left|\dot{\pi}_{L}-(\dot{H} / H) \pi_{L}\right|^{2}+\dot{H} c_{L}^{2} k^{2}\left|\pi_{L}\right|^{2}\right] .
\end{aligned}
$$

The mode functions for tensor fluctuations to first order in slow-roll are,

$$
\gamma_{k}(\tau)=(-\tau)^{3 / 2+\epsilon_{c}} \frac{H_{c}\left(1-\epsilon_{c}\right)}{M_{P}} \sqrt{\frac{\pi}{2}}\left(-\tau_{c}\right)^{-\epsilon_{c}} e^{\frac{i \pi}{2}\left(\nu_{T}+\frac{1}{2}\right)} H_{\nu_{T}}^{(1)}(-k \tau), \quad \nu_{T} \simeq \frac{3}{2}+\epsilon_{c}-\frac{4}{3} c_{T, c}^{2} \epsilon_{c}
$$

where $c_{T}$ and $c_{L}$ are the transvere and longitudinal propagation speed, $H^{(1)}$ is a Hankel function, and the subscript "c" indicates quantities evaluated at some reference time $\tau_{c}$, chosen here as the horizon exit time of the longest modes relevant for observations.

For curvature fluctuations, the mode functions to first order in slow-roll are more involved so we report for simplicity their super-horizon limit $(-k \tau \rightarrow 0)$,

$$
\zeta_{k}(\tau)=\left(\frac{\tau}{\tau_{c}}\right)^{\frac{4}{3} c_{T, c}^{2} \epsilon_{c}}\left(-c_{L, c} k \tau_{c}\right)^{c_{L, c}^{2} \epsilon_{c}-5 s_{c} / 2-\eta_{c} / 2}\left(\frac{H_{c}}{\sqrt{4 \epsilon_{c}} M_{P} c_{L, c}^{5 / 2} k^{3 / 2}}+\mathcal{O}\left(\epsilon^{1 / 2}\right)\right)
$$

where $s \equiv \dot{c}_{L} / H c_{L}$. See Ref. [59] for further details.

Once the wave functions are obtained, it is straightforward to calculate the power spectra $^{13}$ at late times for scalar and tensor modes, they have the following expressions:

$$
P_{\zeta}(k)=\frac{H_{c}^{2}}{4 \epsilon_{c} c_{L, c}^{5} M_{P}^{2}} \frac{1}{k^{3}} \frac{\left(\tau / \tau_{c}\right)^{8 c_{L, c}^{2} \epsilon_{c} / 3}}{\left(-c_{L, c} k \tau_{c}\right)^{5 s_{c}-2 c_{L, c}^{2} \epsilon_{c}+\eta_{c}}}, \quad P_{\gamma}(k)=\frac{H_{c}^{2}}{M_{P}^{2}} \frac{1}{k^{3}} \frac{\left(\tau / \tau_{c}\right)^{8 c_{T, c}^{2} \epsilon_{c} / 3}}{\left(-k \tau_{c}\right)^{-2 c_{L, c}^{2} \epsilon_{c}}} .
$$

\footnotetext{
${ }^{12}$ These differences appear in many facets; e.g., in solid inflation (SI) the physical clock is entirely due to the metric, in SI there are no adiabatic fluctuations, etc..

${ }^{13}$ In SI the gauge-invariant variables $\mathcal{R}$ and $\zeta$ are not equal, not even at late times. They will be after reheating but one needs to choose which quantities to focus on: $\langle\zeta \zeta \ldots\rangle$ or $\langle\mathcal{R} \mathcal{R} \ldots\rangle$. The choice is decided by the fact that in this model $\langle\zeta \zeta\rangle$ must be continuous at reheating.
} 
The spectral indexes for scalars and tensors,

$$
n_{S}-1 \simeq 2 \epsilon_{c} c_{L, c}^{2}-5 s_{c}-\eta_{c}, \quad n_{T} \simeq 2 c_{L, c}^{2} \epsilon_{c},
$$

can be read directly from the expressions above. Note that the tensor tilt is blue. The tensor-to-scalar ratio then has the form:

$$
r \sim \epsilon c_{L}^{5}
$$

For the estimates in Section 5, we use the slow-roll $\left(\epsilon_{c} \rightarrow 0\right)$ approximations,

$$
P_{\zeta}(k)=\frac{H_{c}^{2}}{4 \epsilon_{c} c_{L, c}^{5} M_{P}^{2}} \frac{1}{k^{3}}, \quad P_{\gamma}(k)=\frac{H_{c}^{2}}{M_{P}^{2}} \frac{1}{k^{3}},
$$

for these power spectra.

\subsection{Violations of the consistency conditions}

The squeezed limit of the scalar bispectrum is,

$$
\mathcal{B}_{\zeta \zeta \zeta}\left(k_{L}, k_{S}, k_{S}\right)=-\frac{20}{9} \frac{F_{Y}}{F} \frac{1}{c_{L}^{2} \epsilon}\left(1-3 \cos ^{2} \theta\right) P_{\zeta}\left(k_{L}\right) P_{\zeta}\left(k_{S}\right)
$$

where $\theta$ is the angle between $\vec{k}_{L}$ and $\vec{k}_{S}$. Eq. (4.16) manifestly violates the consistency conditions. The shape of non-Gaussianity for solid inflation has a very small overlap with the local template. However if one, for lack of a better option, relies on Planck $f_{N L}^{\text {local }}$ findings in order to constrain the parameters in Eq. (4.16), then it is safer to assume $F_{Y} \ll F$.

The tensor-scalar-scalar bispectrum reads [67],

$$
\mathcal{B}_{\gamma \zeta \zeta}\left(k_{L}, k_{S}, k_{S}\right)=-\frac{10}{9} \frac{F_{Y}}{F} \frac{1}{c_{L}^{2} \epsilon} P_{\gamma}\left(k_{L}\right) P_{\zeta}\left(k_{S}\right) \simeq-\frac{5}{18} \frac{F_{Y}}{F} \frac{1}{c_{L}^{7} \epsilon^{3}} \frac{H^{4}}{M_{P}^{4}} \frac{1}{k_{L}^{3} k_{S}^{3}} .
$$

Notice that, unless $\left(F_{Y} / F\right)\left(c_{L}^{2} \epsilon\right)^{-1}=-27 / 20$, Eq. (4.17) violates the consistency condition, Eq. (3.16). The bounds to be aware of at this stage are the one in Eq. (4.5) and the ones resulting from the luminality condition on both $c_{T}$ and $c_{L}$, which are related by $c_{T}^{2}=$ $(3 / 4)\left[1+c_{L}^{2}-(2 / 3) \epsilon+(1 / 3) \eta\right]$, to all orders in $\epsilon$ and $\eta$.

\section{Observational signatures}

Primordial scalar perturbations give rise to temperature fluctuations in the CMB and to mass-density perturbations in the late Universe. These late-time mass-density perturbations can be mapped with some precision through their effects on the galaxy distribution, once the effects of galaxy-bias are taken into account, and they can also be mapped through weak gravitational lensing. The effects of tensor metric perturbations can be observed through measurements of CMB fluctuations, and in particular, through measurements of the B mode of the CMB polarization. The effects of tensor metric perturbations may also some day be seen in direct gravitational-wave searches [71-78], but these observations will map only short-wavelength tensor modes. Some of the lensing/CMB/large-scale-structure observations discussed in the Introduction may some day map larger-scale tensor modes, but those measurements are some way in the future. Even these measurements will probably not forward to 
directly map the three-dimensional primordial tensor-perturbation field at the largest scales that we will encounter shortly.

The correlations of primordial tensor perturbations with primordial scalar perturbations can, however, have observational consequences for the mass distribution, even if the tensor perturbation cannot be detected directly. In the absence of a tensor-scalar-scalar bispectrum (and in the approximation that the scalar-scalar-scalar bispectrum is small), the primordial scalar perturbation is Gaussian and statistically isotropic. The tensor-scalar-scalar bispectrum will, however, induce an apparent local departure from statistical isotropy [26-28, 40] and a characteristic non-Gaussian four-point function [29, 37, 38] in primordial perturbations. These effects can be sought in the CMB and in large-scale structure. The depature from statistical isotropy arises primarily from gravitational waves of wavelengths larger than the galaxy-survey size, while the non-Gaussian effects may arise from gravitational waves of wavelengths comparable to the survey size. Departures from statistical isotropy are therefore expected to be most significant, relative to smaller-scale non-Gaussianity, for models where the bispectrum peaks dramatically in the squeezed limit. As we will see, this is what happens for non-attractor inflation, and so we will not work out the smaller-scale non-Gaussianity expected in this model. The k-dependence of the tensor-scalar-scalar bispectrum for solid inflation is, as we will see, closer to the behavior familiar from SFSR, and so we will work out constraints and forecasts for the observability of the small-scale non-Gaussian effects induced in the mass distribution by solid inflation.

\subsection{Local Power Quadrupole}

Here we calculate the local power quadrupole, the observable that follows from the squeezed limit of the tensor-scalar-scalar bispectrum. We begin by summarizing the main results derived above.

We focus in this work on the observable consequences of this bispectrum in the squeezed limit, $k_{L} \ll k_{S}$, where $k_{L} \equiv k_{1}$ and $k_{S} \equiv k_{2} \simeq k_{3}$. In both models we first account for a contribution of the form given in Eq. (3.16) in the squeezed limit. This is the contribution that arises from the consistency condition between the squeezed-limit bispectrum and the scalar and tensor power spectra $P_{\gamma}\left(k_{L}\right)$ and $P_{\zeta}\left(k_{S}\right)$, respectively. This part of the bispectrum gives rise to an infrared-divergent contribution to the local power quadrupole moment that is then cancelled by a similarly infrared-divergent late-time effect $[28,55]$ leaving a small, but nonzero and observable (at least in principle), local power quadrupole [28, 40].

What we are interested in here, though, is the part of the bispectrum that violates ${ }^{14}$ the consistency condition. For non-attractor inflation, this was found to be [cf., Eq. (3.17)],

$$
\mathcal{B}_{\text {na }, \text { ck }}\left(k_{L}, k_{S}, k_{S}\right)=\frac{297}{32} \frac{H_{*}^{4}}{M_{P}^{4}}\left(\frac{1}{\epsilon_{*} c_{s}}\right)\left(\frac{1}{k_{L}^{3} k_{S}^{3}}\right) \epsilon_{*} \frac{1}{\left(\tau_{*} c_{s} k_{S}\right)^{6}}\left(\frac{k_{L}}{c_{s} k_{S}}\right)^{2} .
$$

For solid inflation it is, from Eq. (4.17),

$$
\mathcal{B}_{\mathrm{si}, \not c}\left(k_{L}, k_{S}, k_{S}\right)=-\frac{5}{18}\left(\frac{F_{Y}}{F} \frac{1}{c_{L}^{2} \epsilon}+\frac{27}{20}\right) \frac{1}{c_{L}^{5} \epsilon^{2}} \frac{H^{4}}{M_{P}^{4}} \frac{1}{k_{L}^{3} k_{S}^{3}} .
$$

The existence of gravitational waves with wavelengths long compared with the distances over which observations are performed (e.g., for the CMB, our observable horizon) gives rise

\footnotetext{
${ }^{14}$ As mentioned, for non-attractor inflation the violation is to be understood in the sense of Subsection 2.3.
} 
to an apparent local departure from statistical isotropy. In other words, the rms amplitudes of Fourier modes of the same wavenumber but different directions may differ. An individual Fourier mode $\gamma_{p}\left(\vec{k}_{L}\right)$ of the tensor field ${ }^{15}$ gives rise to a local matter, or curvature, power spectrum,

$$
\left.P_{\zeta}\left(\vec{k}_{S}\right)\right|_{\gamma_{p}\left(\vec{k}_{L}\right)}=P_{\zeta}\left(k_{S}\right)\left[1+\mathcal{Q}_{i j}^{p}\left(\vec{k}_{L}\right) \hat{k}_{S}^{i} \hat{k}_{S}^{j}\right],
$$

with power quadrupole,

$$
\mathcal{Q}_{i j}^{p}\left(\vec{k}_{L}\right)=\frac{\mathcal{B}_{c k}\left(k_{L}, k_{S}, k_{S}\right)}{P_{\gamma}\left(k_{L}\right) P_{\zeta}\left(k_{S}\right)} \gamma_{i j}^{p}\left(\vec{k}_{L}\right)
$$

where $\mathcal{B}_{c k}\left(k_{L}, k_{S}, k_{S}\right)$ is the consistency-condition-violating part of the tensor-scalar-scalar bispectrum. The observed power quadrupole is then obtained by summing over both gravitationalwave polarizations $p=\{+, \times\}$ and Fourier wavevectors $\vec{k}_{L}$.

The theory then predicts that this locally observed power quadrupole has variance,

$$
\overline{\mathcal{Q}^{2}} \equiv \frac{8 \pi}{15}\left\langle\mathcal{Q}_{i j} \mathcal{Q}^{i j}\right\rangle=\frac{16}{15 \pi} \int_{k_{L}^{\min }}^{k_{S}^{\min }} k_{L}^{2} d k_{L}\left[\frac{\mathcal{B}_{c k}\left(k_{L}, k_{S}, k_{S}\right)}{P_{\gamma}\left(k_{L}\right) P_{\zeta}\left(k_{S}\right)}\right]^{2} P_{\gamma}\left(k_{L}\right)
$$

Here, the upper limit of integration, $k_{S}^{\mathrm{min}}$, is the smallest wavenumber probed by the observations. The lower limit, $k_{L}^{\mathrm{min}}$, corresponds to the longest-wavelength gravitational-wave mode produced during inflation.

Using $P_{\gamma}(k)=\left(1 / k^{3}\right)\left(H_{*} / M_{P}\right)^{2}$, the result for non-attractor inflation is

$$
\overline{\mathcal{Q}_{\text {na }}^{2}}=\frac{64}{15 \pi}\left(\frac{297}{32}\right)^{2}\left(\frac{H_{*}}{M_{P}}\right)^{2}\left(\frac{k_{S}^{\min }}{c_{S} k_{S}}\right)^{4} \frac{\epsilon_{*}^{2}}{\left(c_{s} k_{S} \tau_{*}\right)^{12}} .
$$

The $k_{L}^{2}$ dependence of $\mathcal{B} / P_{\gamma}$ assures that the result does not depend on the infrared cutoff $k_{L}^{\mathrm{min}}$. The falloff of the power quadrupole with increasing $k_{S}$ is so steep that the observability of the signal will depend almost entirely on the sensitivity to a power quadrupole on the very largest scales. Roughly speaking, the observational upper limit will be $\overline{\mathcal{Q}_{n a}^{2}} \lesssim 1$ for $k_{S} \sim H_{0}$, the Hubble parameter today. This translates into a bound $\tau_{*} \gtrsim H_{0}^{-1}$, which, given the very strong dependence of the quadrupole on $k_{S}$, is fairly insensitive to other model parameters. We thus infer that the absence of any grotesque departure of the components of the CMB quadrupole from statistical isotropy tells us that a non-attractor phase of inflation must have ended no later than the time that our current Universe exited the horizon during inflation.

For solid inflation, the result is

$$
\overline{\mathcal{Q}_{\mathrm{si}}^{2}}=\frac{64}{15 \pi}\left[\frac{5}{9}\left(\frac{F_{Y}}{F c_{L}^{2} \epsilon}+\frac{27}{20}\right)\right]^{2} \frac{H_{c}^{2}}{\epsilon^{2} M_{P}^{2}} \ln \left(\frac{k_{S}^{\min }}{k_{L}^{\min }}\right) \equiv A \ln \left(\frac{k_{S}^{\min }}{k_{L}^{\min }}\right),
$$

which defines the prefactor $A$. In this case, the power quadrupole diverges logarithmically as $k_{L}^{\min } \rightarrow 0$, implying sensitivity of the observable to very-long-wavelength modes, something that does not arise for the part of the bispectrum that satisfies the consistency condition. The observation that $\overline{\mathcal{Q}_{\text {na }}^{2}} \lesssim 1$ for $k_{S} \sim H_{0}$ then implies an upper limit $A\left|\ln \left(k_{L}^{\min } H_{0}^{-1}\right)\right| \lesssim 1$.

\footnotetext{
${ }^{15}$ Any collection of modes with wavelengths much longer than the horizon will be undistinguishable from one another within any single horizon. One effectively has a single, long-wavelength, mode.
} 


\subsection{Clustering fossils}

Here we consider the the characteristic non-Gaussian four-point correlations in the scalar perturbation induced by coupling to tensor modes. Ref. [38] provides a recipe for measuring these correlations with a galaxy survey (or other tracer of the three-dimensional mass distribution) and estimates the detectability of the signal for single-field slow-roll inflation. We first consider the case with the primordial bispectrum obeying the SFSR consistency relation including the late-time effects of tensor-scalar coupling and projection effect, then move on to the case with solid inflation and calculate the signal-to-noise ratio for measuring the signature of clustering fossils.

\subsubsection{Clustering fossils with the consistency relation}

The consistency relation dictates that the tensor-scalar-scalar bispectrum takes the form in Eq. (3.16) in the squeezed limit. This bispectrum implies that in the presence of a Fourier mode $\gamma_{p}\left(\vec{k}_{L}\right)$ of the tensor perturbation, the correlation between two scalar-perturbation modes $\zeta\left(\vec{k}_{1}\right)$ and $\zeta\left(\vec{k}_{2}\right)$ is [38],

$$
\left.\left\langle\zeta\left(\vec{k}_{1}\right) \zeta\left(\vec{k}_{2}\right)\right\rangle\right|_{\gamma_{p}\left(\vec{k}_{L}\right)}=\delta_{\vec{k}_{1}+\vec{k}_{2}}^{D} P_{\zeta}\left(k_{1}\right)-\frac{1}{2} \delta_{\vec{k}_{L}+\vec{k}_{1}+\vec{k}_{2}}^{D} \frac{\mathrm{d} \ln P_{\zeta}\left(k_{1}\right)}{\mathrm{d} \ln k_{1}} P_{\zeta}\left(k_{1}\right) \gamma_{p}\left(\vec{k}_{L}\right) \hat{\varepsilon}_{i j}\left(\hat{k}_{L}\right) \hat{k}_{1}^{i} \hat{k}_{2}^{j}
$$

where we use the shorthand $\delta_{\vec{k}}^{D} \equiv(2 \pi)^{3} \delta_{D}(\vec{k})$. There is now a new term, in addition to the usual power spectrum, that correlates different Fourier modes of the scalar perturbation. This off-diagonal correlator can be understood as a local rescaling, $k^{2} \rightarrow\left(\delta_{i j}+\gamma_{i j}\left(\vec{k}_{K}\right)\right) k^{i} k^{j}$, of the wavevector.

Until inflation ends, all relevant density modes are outside the horizon with the local correlation function frozen with the form of Eq. (5.8). After inflation ends, density modes continuously come inside the horizon and evolve under the influence of the long-wavelength tensor field. This yields a local density contrast,

$$
\left.\delta\left(\vec{k}_{S}\right)\right|_{\gamma\left(\vec{k}_{L}\right)}=2 T_{\delta}\left(k_{S}\right)\left[1-\left(\frac{1}{2} \frac{\mathrm{d} \ln T_{\delta}\left(k_{S}\right)}{\mathrm{d} \ln k_{S}}+S_{N}\left(k_{L}\right)\right) \gamma^{i j}\left(\vec{k}_{L, i}\right) \hat{k}_{S, i} \hat{k}_{S, j}\right] \zeta\left(\vec{k}_{S}\right),
$$

where $T_{\delta}\left(k_{S}\right)$ and $T_{\gamma}\left(k_{L}\right)$ are the transfer functions for, respectively, the density field and gravitational wave, and

$$
S_{N}\left(k_{L}\right) \simeq \frac{3}{5}\left[1-\exp \left(-\frac{5}{42} k_{L}^{2} \eta^{2}\right)\right]
$$

encodes the dynamical influence of the long-wavelength tensor mode $\gamma_{p}\left(\vec{k}_{L}\right)$ on the evolution of the small-scale scalar mode [28]. At large scales $\left(k_{L} \rightarrow 0\right)$, the function $S_{N}\left(k_{L}\right)$ vanishes; this obeys the causality demand that there is no influence from super-horizon tensor modes on the evolution of subhorizon scalar modes. On small scales $\left(k_{L} \gg H_{0}\right)$, the function $S_{N}\left(k_{L}\right)$ asymptotes to $3 / 5$. This then partially cancels the primordial off-diagonal correlation in Eq. (5.8) to yield an observed density field that satisfies,

$$
\left.\left\langle\delta\left(\vec{k}_{1}\right) \delta\left(\vec{k}_{2}\right)\right\rangle\right|_{\gamma_{p}\left(\vec{k}_{L}\right)} \simeq \delta_{\vec{k}_{1}+\vec{k}_{2}}^{D} P_{\delta}\left(k_{1}\right)-\delta_{\vec{k}_{1}+\vec{k}_{2}+\vec{k}_{L}}^{D}\left[\frac{1}{2} \frac{\mathrm{d} \ln P_{\delta}(k)}{\mathrm{d} \ln k}+2 S_{N}\left(k_{L}\right)\right] P_{\delta}\left(k_{1}\right) \gamma^{i j}\left(\vec{k}_{L}\right) \hat{k}_{1}^{i} \hat{k}_{2}^{j} .
$$


Finally, with the linear-bias parameter $b_{g}$, the observed galaxy density contrast is given in terms of the intrinsic matter density contrast and the projection at the location of galaxies and the line-of-sight as $[16,28,39,79]$ :

$$
\delta_{g}^{\mathrm{obs}}=b_{g}\left[\delta+\Delta x^{i} \partial_{i} \delta+\left(b_{e} H \Delta t+\partial_{i} \Delta x^{i}\right) \delta\right] \simeq b_{g} \delta-\frac{1}{2} b_{g} T_{\gamma} \gamma^{i j} x_{j} \partial_{i} \delta
$$

with the temporal and spatial displacement $\left(\Delta t\right.$ and $\left.\Delta x^{i}\right)$ of galaxies due to light deflection. The dominant projection effect comes from large scales $k_{L} \ll H_{0}$ where $\Delta t$ vanishes and $\Delta x^{i}=-T_{\gamma} \gamma^{i j} x_{j} / 2$ [16]. Including projection effects [28], the observed galaxy-density field thus satisfies,

$$
\begin{aligned}
\left.\left\langle\delta_{g}\left(\vec{k}_{1}\right) \delta_{g}\left(\vec{k}_{2}\right)\right\rangle\right|_{\gamma_{p}\left(\vec{k}_{L}\right)} \simeq & \delta_{\vec{k}_{1}+\vec{k}_{2}}^{D} P_{g}\left(k_{1}\right) \\
& -\delta_{\vec{k}_{1}+\vec{k}_{2}+\vec{k}_{L}}^{D}\left[\frac{1}{2}\left(1-T_{\gamma}\right) \frac{\mathrm{d} \ln P_{\delta}\left(k_{1}\right)}{\mathrm{d} \ln k_{1}}+2 S_{N}\left(k_{L}\right)\right] P_{g}\left(k_{1}\right) \gamma^{i j}\left(\vec{k}_{L}\right) \hat{k}_{1}^{i} \hat{k}_{2}^{j} .
\end{aligned}
$$

This equation corrects the clustering-fossil result in Ref. [38] to take into account the cancellation of the infrared divergence from the initial bispectrum in these observables from projection effects $[28,55]$ leaving an observable $\mathcal{O}\left(k_{L}^{2}\right)$ clustering fossil [28].

The inclusion here of late-time effects revises the result in Fig. 2 of Ref. [38] for bispectra that satisfy the consistency condition. After taking these effects into account, the $3 \sigma$ detection limits in Eq. (10) and Fig. 2 of Ref. [38] are increased by a factor,

$$
\left(\frac{1}{2} \frac{\mathrm{d} \ln P_{\delta}\left(k_{1}\right)}{\mathrm{d} \ln k_{1}}\right)^{2}\left[\frac{1}{2}\left(1-T_{\gamma}\right) \frac{\mathrm{d} \ln P_{\delta}\left(k_{1}\right)}{\mathrm{d} \ln k_{1}}+2 S_{N}(K)\right]^{-2} \simeq 25
$$

for $K \gtrsim k_{H}$.

\subsubsection{Clustering fossils in solid inflation}

The tensor-scalar-scalar correlator can be used to estimate the amplitude of tensor modes. One can construct an optimal variance estimator [38] for the tensor power spectrum, with variance $\sigma_{\gamma}$ given by

$$
\sigma_{\gamma}^{-2}=\frac{1}{2} \sum_{\vec{k}_{L}, p}\left[k_{L}^{3} P_{p}^{n}\left(k_{L}\right)\right]^{-2}
$$

where $P_{p}^{n}$ is the noise power spectrum, defined as

$$
P_{p}^{n}\left(k_{L}\right)=\left[\sum_{\vec{k}_{S}} \frac{\left|\mathcal{B}_{\mathrm{cc}}\left(k_{L}, k_{S},\left|\vec{k}_{L}-\vec{k}_{S}\right|\right) \epsilon_{i j}^{p} \hat{k}_{S}^{i} \hat{k}_{L S}^{j}\right|^{2}}{2 V P_{\gamma}^{2}\left(k_{L}\right) P_{\zeta}^{t o t}\left(k_{S}\right) P_{\zeta}^{t o t}\left(\left|\vec{k}_{L}-\vec{k}_{S}\right|\right)}\right]^{-1} .
$$

Above $\hat{k}_{L S} \equiv\left(\vec{k}_{L}-\vec{k}_{S}\right) /\left|\vec{k}_{L}-\vec{k}_{S}\right|$, $P_{\zeta}^{\text {tot }}$ is the total measured scalar power spectrum (i.e. including signal and noise) and $V$ is the total volume of the survey. Notice that the variance in Eq. (5.15) is inversely proportional to the variance of the quadrupole. As a result, for a given survey size, the larger the amplitude of the quadrupole, the larger the minimum amplitude of tensor modes that one is able to probe. 
Given the similarity of the $k_{L}$ and $k_{S}$ dependences of scalar and tensor power spectra and tensor-scalar-scalar bispectrum in solid inflation with those of SFSR inflation

$$
\mathcal{B}_{\mathrm{si}, \mathrm{cc}}\left(k_{L}, k_{S}, k_{S}\right)=-\frac{20}{18 \epsilon}\left(\frac{F_{Y}}{F} \frac{1}{c_{L}^{2} \epsilon}+\frac{27}{20}\right) P_{\zeta}\left(k_{S}\right) P_{\gamma}\left(k_{L}\right) \equiv-\frac{3}{2} \frac{\mathcal{R}}{\epsilon} P_{\zeta}\left(k_{S}\right) P_{\gamma}\left(k_{L}\right)
$$

the SFSR results in Ref. [38] are easily adapted to solid inflation. Ref. [38] shows that for SFSR inflation (and neglecting late-time effects), the smallest tensor amplitude $A_{\gamma}$, defined by $P_{\gamma}\left(k_{L}\right)=A_{\gamma} k_{L}^{-3}$, detectable at the $\gtrsim 3 \sigma$ level is $\sim 300\left(k_{\max } / k_{\min }\right)^{-3}$, where $k_{\min }$ and $k_{\max }$ are the minimum and maximum wavenumbers, respectively, probed by a given galaxy survey. Detection of SFSR tensors near the maximum amplitude, $A_{\gamma} \simeq 2 \times 10^{-9}$, currently allowed requires $k_{\max } / k_{\text {min }} \gtrsim 5000$, beyond the reach of galaxy surveys but perhaps within reach of future 21-cm mapping experiments.

In solid inflation there is a similar prediction for this galaxy four-point correlation function that arises from the part of the tensor-scalar-scalar bispectrum that satisfies the consistency condition. There is then, however, an additional contribution from the consistencycondition-violating part that is $\mathcal{R} / \epsilon$ times the naive (neglecting late-time effects) prediction given in Ref. [38] from the consistency condition. This number must exceed $1 / 5$ if the ccviolating four-point signal is to dominate the cc-preserving signal. If the tensor-to-scalar ratio is indeed as large as $r \sim 0.1$, then a model with $\mathcal{R} / \epsilon \gtrsim 27$ will give a detectable signal in a galaxy survey, like EUCLID, with $k_{\max } / k_{\min } \simeq 750$. A model with $\mathcal{R} / \epsilon \gtrsim 1.5$ will be detectable in a 21 -cm survey that maps a volume with $k_{\max } / k_{\min } \simeq 5000$.

As seen above, the quadrupole constrains $\left(H_{c} \mathcal{R} / \epsilon M_{P}\right)^{2}\left|\ln \left(k_{L}^{\text {min }} H_{0}^{-1}\right)\right| \lesssim 1$. Thus, for example, if $r \sim 0.1$, the quadrupole constraint is (taking the $\log$ to be $\sim 10) \mathcal{R} / \epsilon \lesssim 10^{4}$. We thus see that it is easily possible to have a solid-inflation model consistent with the quadrupole constraint and which will still have a clustering-fossil signature large enough to be detectable in forthcoming large-scale-structure surveys. In particular, this range of values for $\mathcal{R} / \epsilon$ corresponds to a region in the parameter space of the theory where the parameters naturally lie $^{16}$. In fact, they may even be conceivably large enough to be detectable with existing data!

\section{Conclusions}

In this paper we have studied the squeezed limit of the tensor-scalar-scalar bispectrum induced during inflation. We reviewed how the consistency condition found in Ref. [42] relating this bispectrum to the scalar and tensor power spectra generalizes to any single-clock inflation model. We then computed the TSS correlator in non-attractor inflation. There the decaying mode (which is ordinarily negligible in single-clock models) causes a departure from the single-clock dynamics. The non-attractor inflationary phase, which is followed by a more traditional attractor phase, leaves an important imprint on the three-point function in the squeezed limit; specifically, it manifests itself at quadratic order in the soft (tensor) momentum. In solid inflation, the anisotropic stress of the medium leads to a direct violation of the consistency conditions.

The TSS bispectrum in non-attractor inflation can give rise to an apparent quadrupolar departure from statistical isotropy (SI) in large-scale structure, with the SI violation most

\footnotetext{
${ }^{16}$ As discussed, the ratio $F_{Y} / F$ needs to be no larger than unity but that leaves plenty of room for an intriguing value of $\mathcal{R} / \epsilon$ to be accommodated.
} 
significant at the largest scales. The consistency of the CMB quadrupole with SI constrains the transition from the non-attractor to attractor phase to occur before the time that the current observable Universe exited the horizon during inflation. The very rapid decay of the SI violation suggests that there will be no further observable consequences of the squeezedlimit TSS on smaller scales.

The effects of the TSS bispectrum from solid inflation are distributed much more evenly among different distance scales. Thus, it is conceivable that there may be clustering fossils of the type discussed in Ref. [38] in large-scale structure of a magnitude that could be detectable with forthcoming survey, and possibly even with current data. Heuristically, the effects of the anisotropic medium that fills the Universe during inflation may be written in the distribution of galaxies today! We thus encourage the pursuit of such signatures.

Here we have shown only that these effects may occur with appreciable magnitudes in solid inflation. More work must be done to map out the parameter space of solid-inflation models (as well as related models, like gauge-flation or chromo-natural inflation [89-93]) in which such signatures may arise. It will also be interesting to explore the magnitude of effects induced by the TSS bispectrum in other models of inflation that may violate the consistency conditions.

\section{Acknowledgments}

It is a pleasure to thank Junpu Wang and Lasha Berezhiani for useful discussions, and Razieh Emami and Hassan Firouzjahi for valuable correspondence and comments on an earlier version of the manuscript. MK and DJ were supported by NSF Grant No. 0244990 and the John Templeton Foundation. ED acknowledges partial support from the DOE grant DE-SC0011842 at the University of Minnesota. MF is supported in part by DOE DESC0010600. ED and MF would like to thank the Cosmology group at Johns Hopkins Physics and Astronomy Department for very warm hospitality whilst parts of this work were being completed.

\section{A Zeroth and first order CCS}

For completeness, we report here from [52] (see also [47]) the SSS and TSS consistency conditions up to linear order in the soft momentum, which is their most familiar form. For the scalar one has

$$
\begin{aligned}
& \frac{\left\langle\zeta_{\vec{q}} \zeta_{\vec{p}} \zeta_{-\vec{q}-\vec{p}}\right\rangle^{\prime}}{P_{\zeta}(q)}= \\
& \quad-\left(3+p_{k} \frac{\partial}{\partial p_{k}}\right) P_{\zeta}(p)-\frac{1}{2} q_{k}\left(6 \frac{\partial}{\partial p_{k}}-p_{k} \frac{\partial^{2}}{\partial p_{a} \partial p_{a}}+2 p_{a} \frac{\partial^{2}}{\partial p_{a} \partial p_{k}}\right) P_{\zeta}(p)+\mathcal{O}\left(q^{2}\right) .
\end{aligned}
$$

while the TSS reads

$$
\begin{aligned}
& \frac{\left\langle\gamma_{\vec{q}}^{i j} \zeta_{\vec{p}} \zeta_{-\vec{q}-\vec{p}}\right\rangle^{\prime}}{P_{\gamma}(q)}= \\
& \quad-\frac{1}{2} \hat{P}^{i j k \ell}(\hat{q}) p_{k} \frac{\partial}{\partial p_{\ell}} P_{\zeta}(p)+\frac{1}{4} \hat{P}^{i j k \ell}(\hat{q}) q_{m}\left(p_{m} \frac{\partial^{2}}{\partial p_{k} \partial p_{\ell}}-2 p_{k} \frac{\partial^{2}}{\partial p_{\ell} \partial p_{m}}\right) P_{\zeta}(p)+\mathcal{O}\left(q^{2}\right) .
\end{aligned}
$$




\section{B The tensor-scalar-scalar action}

The action at third order in the perturbations $\gamma \zeta \zeta$ has the form $[42,80,81]$,

$\mathcal{S}_{\gamma \zeta^{2}}=\int d t d^{3} x\left\{-2 \frac{a}{H} \gamma_{i j} \partial_{i} \dot{\zeta} \partial_{j} \zeta-a \gamma_{i j} \partial_{i} \zeta \partial_{j} \zeta-\frac{1}{2} a^{3}\left(3 \zeta-\frac{\dot{\zeta}}{H}\right) \dot{\gamma}_{i j} \partial_{i} \partial_{j} \psi+\frac{1}{2} a^{3} \partial_{k} \gamma_{i j} \partial_{i} \partial_{j} \psi \partial_{l} \psi\right\}$,

where $\psi$ represents the shift function of the metric, Eq. (3.4), $N^{i}=\partial_{i} \psi$, with $\psi=-\zeta / a H+\chi$ and $\partial^{2} \chi=\left(\epsilon / c_{s}^{2}\right) \dot{\zeta}$. Multiple partial integrations can be performed to bring Eq. (B.1) to a simpler form,

$$
\begin{aligned}
\mathcal{S}_{\gamma \zeta^{2}} & =\int d t d^{3} x\left\{\epsilon a \gamma_{i j} \partial_{i} \zeta \partial_{j} \zeta+\frac{1}{4} a^{3} \partial^{2} \gamma_{i j} \partial_{i} \chi \partial_{j} \chi+\frac{1}{2} \epsilon a^{3} \dot{\gamma}_{i j} \partial_{i} \zeta \partial_{j} \chi\right. \\
& \left.\left.+f(\zeta, \gamma) \frac{\delta L}{\delta \zeta}+f_{i j}(\zeta, \gamma) \frac{\delta L}{\delta \gamma_{i j}}\right\}\right],
\end{aligned}
$$

where the last two terms are proportional to the equations of motion for $\zeta$ and $\gamma$ and can be therefore eliminated by a field redefinition

$$
\zeta=\zeta_{n}+f\left(\zeta_{n}, \tilde{\gamma}_{i j}\right), \quad \gamma_{i j}=\tilde{\gamma}_{i j}+f_{i j}\left(\zeta_{n}, \tilde{\gamma}_{i j}\right) .
$$

The complete expressions for the functions $f$ and $f_{i j}$ are [42, 80, 82],

$$
\begin{aligned}
f\left(\zeta_{n}, \tilde{\gamma}_{i j}\right) & \equiv \frac{1}{2} \frac{\ddot{\phi}}{\dot{\phi} H} \zeta_{n}^{2}+\frac{\epsilon}{2} \zeta_{n}^{2}+\frac{1}{H} \dot{\zeta}_{n} \zeta_{n}-\frac{1}{4} \frac{1}{a^{2} H^{2}}\left(\partial \zeta_{n}\right)^{2}+\frac{1}{4} \frac{1}{a^{2} H^{2}} \partial^{-2} \partial_{i} \partial_{j}\left(\partial_{i} \zeta_{n} \partial_{j} \zeta_{n}\right) \\
& +\frac{1}{2} \frac{1}{H} \partial_{i} \chi_{n} \partial_{j} \zeta_{n}-\frac{1}{2} \frac{1}{H} \partial^{-2} \partial_{i} \partial_{j}\left(\partial_{i} \chi_{n} \partial_{j} \zeta_{n}\right)-\frac{1}{4} \frac{1}{H} \dot{\tilde{\gamma}}_{i j} \partial^{-2} \partial_{i} \partial_{j} \zeta_{n} \\
f_{i j}\left(\zeta_{n}, \tilde{\gamma}_{i j}\right) & \equiv \frac{1}{H} \dot{\tilde{\gamma}}_{i j} \zeta_{n}-\frac{1}{a^{2} H^{2}} \partial_{i} \zeta_{n} \partial_{j} \zeta_{n}+\frac{1}{H}\left(\partial_{i} \chi_{n} \partial_{j} \zeta_{n}+\partial_{j} \chi_{n} \partial_{i} \zeta_{n}\right) .
\end{aligned}
$$

We report below the contributions to the tensor-scalar-scalar bispectrum from the field redefinitions. The leading-order contributions (in powers of $H / M_{P}$ ) have the form,

$$
\mathcal{B}\left(k_{1}, k_{2}, k_{3}\right)_{F R}=\mathcal{B}_{\left[\zeta \rightarrow \tilde{\gamma} \zeta_{n}\right]}\left(k_{1}, k_{2}, k_{3}\right)+\mathcal{B}_{\left[\gamma \rightarrow \zeta_{n}^{2}\right]}\left(k_{1}, k_{2}, k_{3}\right)
$$

The first contribution in Eq. (B.6) arises from Eq. (B.4), specifically from the expression of the curvature fluctuations as a scalar-tensor convolution,

$$
\zeta \rightarrow-\frac{1}{4} \frac{1}{H} \dot{\tilde{\gamma}}_{i j} \partial^{-2} \partial_{i} \partial_{j} \zeta_{n}
$$

The second contribution is due to Eq. (B.5). In particular, it comes from the redefinition of a tensor fluctuation in terms of the convolution of two scalars,

$$
\gamma_{i j} \rightarrow-\frac{1}{a^{2} H^{2}} \partial_{i} \zeta_{n} \partial_{j} \zeta_{n}+\frac{1}{H}\left(\partial_{i} \chi_{n} \partial_{j} \zeta_{n}+\partial_{j} \chi_{n} \partial_{i} \zeta_{n}\right)
$$

In the limit $k_{1} \equiv k_{L} \ll k_{2} \simeq k_{3} \equiv k_{S}$, one finds

$$
\begin{aligned}
& \mathcal{B}_{\left[\zeta \rightarrow \tilde{\gamma} \zeta_{n}\right]}\left(k_{L}, k_{S}, k_{S}\right)=\frac{1}{8} \frac{H_{*}^{4}}{M_{P}^{4}}\left(\frac{1}{\epsilon_{*} c_{s}}\right)\left(\frac{1}{k_{S}^{3} k_{L}^{3}}\right) \frac{\left(k_{S} c_{s} \tau_{0}\right)^{2}}{c_{s}^{2}}\left(\frac{k_{L}}{k_{S}}\right)^{2}, \\
& \mathcal{B}_{\left[\gamma \rightarrow \zeta_{n}^{2}\right]}\left(k_{L}, k_{S}, k_{S}\right)=\frac{1}{8} \frac{H_{*}^{4}}{M_{P}^{4}}\left(\frac{1}{\epsilon_{*} c_{s}}\right)\left(\frac{1}{k_{S}^{3} k_{L}^{3}}\right)\left(\frac{1}{\epsilon_{*}}-2\right) \frac{\left(k_{S} c_{s} \tau_{0}\right)^{2}}{c_{s}^{3}}\left(\frac{k_{L}}{k_{S}}\right)^{3},
\end{aligned}
$$

where $\tau_{0}$ is the time of observation. 


\section{References}

[1] A. H. Guth and S. Y. Pi, "Fluctuations in the New Inflationary Universe," Phys. Rev. Lett. 49, 1110 (1982).

[2] J. M. Bardeen, P. J. Steinhardt and M. S. Turner, "Spontaneous Creation of Almost Scale Free Density Perturbations in an Inflationary Universe," Phys. Rev. D 28, 679 (1983).

[3] S. W. Hawking, "The Development of Irregularities in a Single Bubble Inflationary Universe," Phys. Lett. B 115, 295 (1982).

[4] A. D. Linde, "A New Inflationary Universe Scenario: A Possible Solution of the Horizon, Flatness, Homogeneity, Isotropy and Primordial Monopole Problems," Phys. Lett. B 108, 389 (1982).

[5] V. F. Mukhanov and G. V. Chibisov, "Quantum Fluctuation and Nonsingular Universe. (In Russian)," JETP Lett. 33, 532 (1981) [Pisma Zh. Eksp. Teor. Fiz. 33, 549 (1981)].

[6] L. F. Abbott and M. B. Wise, "Constraints on Generalized Inflationary Cosmologies," Nucl. Phys. B 244, 541 (1984).

[7] V. A. Rubakov, M. V. Sazhin and A. V. Veryaskin, "Graviton Creation in the Inflationary Universe and the Grand Unification Scale," Phys. Lett. B 115, 189 (1982).

[8] R. Fabbri and M. d. Pollock, "The Effect of Primordially Produced Gravitons upon the Anisotropy of the Cosmological Microwave Background Radiation," Phys. Lett. B 125, 445 (1983).

[9] A. A. Starobinsky, "Relict Gravitation Radiation Spectrum and Initial State of the Universe. (In Russian)," JETP Lett. 30, 682 (1979) [Pisma Zh. Eksp. Teor. Fiz. 30, 719 (1979)].

[10] M. Kamionkowski, A. Kosowsky and A. Stebbins, "A Probe of primordial gravity waves and vorticity," Phys. Rev. Lett. 78, 2058 (1997) [astro-ph/9609132].

[11] U. Seljak and M. Zaldarriaga, "Signature of gravity waves in polarization of the microwave background," Phys. Rev. Lett. 78, 2054 (1997) [astro-ph/9609169].

[12] P. A. R. Ade et al. [BICEP2 Collaboration], "Detection of B-Mode Polarization at Degree Angular Scales by BICEP2," Phys. Rev. Lett. 112, 241101 (2014) [arXiv:1403.3985 [astro-ph.CO]].

[13] G. Hinshaw et al. [WMAP Collaboration], "Nine-Year Wilkinson Microwave Anisotropy Probe (WMAP) Observations: Cosmological Parameter Results," Astrophys. J. Suppl. 208, 19 (2013) [arXiv:1212.5226 [astro-ph.CO]].

[14] P. A. R. Ade et al. [Planck Collaboration], "Planck 2013 results. XVI. Cosmological parameters," arXiv:1303.5076 [astro-ph.CO].

[15] S. Dodelson, E. Rozo and A. Stebbins, "Primordial gravity waves and weak lensing," Phys. Rev. Lett. 91, 021301 (2003) [astro-ph/0301177].

[16] F. Schmidt and D. Jeong, "Large-Scale Structure with Gravitational Waves II: Shear," Phys. Rev. D 86, 083513 (2012) [arXiv:1205.1514 [astro-ph.CO]].

[17] L. Dai, M. Kamionkowski and D. Jeong, "Total Angular Momentum Waves for Scalar, Vector, and Tensor Fields," Phys. Rev. D 86, 125013 (2012) [arXiv:1209.0761 [astro-ph.CO]].

[18] N. E. Chisari, C. Dvorkin and F. Schmidt, "Can weak lensing surveys confirm BICEP2 ?," arXiv:1406.4871 [astro-ph.CO].

[19] A. Cooray, M. Kamionkowski and R. R. Caldwell, "Cosmic shear of the microwave background: The Curl diagnostic," Phys. Rev. D 71, 123527 (2005) [astro-ph/0503002].

[20] C. Li and A. Cooray, "Weak Lensing of the Cosmic Microwave Background by Foreground 
Gravitational Waves," Phys. Rev. D 74, 023521 (2006) [astro-ph/0604179].

[21] S. Dodelson, "Cross-Correlating Probes of Primordial Gravitational Waves," Phys. Rev. D 82, 023522 (2010) [arXiv:1001.5012 [astro-ph.CO]].

[22] L. G. Book, M. Kamionkowski and T. Souradeep, "Odd-Parity Bipolar Spherical Harmonics," Phys. Rev. D 85, 023010 (2012) [arXiv:1109.2910 [astro-ph.CO]].

[23] U.-L. Pen, "Gravitational lensing of pre-reionization gas," New Astron. 9, 417 (2004) [astro-ph/0305387].

[24] K. W. Masui and U.-L. Pen, "Primordial gravity wave fossils and their use in testing inflation," Phys. Rev. Lett. 105, 161302 (2010) [arXiv:10,06.4181 [astro-ph.CO]].

[25] L. Book, M. Kamionkowski and F. Schmidt, "Lensing of 21-cm Fluctuations by Primordial Gravitational Waves," Phys. Rev. Lett. 108, 211301 (2012) [arXiv:1112.0567 [astro-ph.CO]].

[26] S. B. Giddings and M. S. Sloth, "Cosmological observables, IR growth of fluctuations, and scale-dependent anisotropies," Phys. Rev. D 84, 063528 (2011) [arXiv:1104.0002 [hep-th]].

[27] L. Dai, D. Jeong and M. Kamionkowski, "Seeking Inflation Fossils in the Cosmic Microwave Background," Phys. Rev. D 87, no. 10, 103006 (2013) [arXiv:1302.1868 [astro-ph.CO]].

[28] L. Dai, D. Jeong and M. Kamionkowski, "Anisotropic imprint of long-wavelength tensor perturbations on cosmic structure," Phys. Rev. D 88, 043507 (2013) [arXiv:1306.3985 [astro-ph.CO]].

[29] S. Brahma, E. Nelson and S. Shandera, "Fossilized Gravitational Wave Relic and Primordial Clocks," Phys. Rev. D 89, 023507 (2014) [arXiv:1310.0471 [astro-ph.CO]].

[30] A. R. Pullen and M. Kamionkowski, "Cosmic Microwave Background Statistics for a Direction-Dependent Primordial Power Spectrum," Phys. Rev. D 76, 103529 (2007) [arXiv:0709.1144 [astro-ph]].

[31] D. Hanson and A. Lewis, "Estimators for CMB Statistical Anisotropy," Phys. Rev. D 80, 063004 (2009) [arXiv:0908.0963 [astro-ph.CO]].

[32] N. E. Groeneboom and H. K. Eriksen, "Bayesian analysis of sparse anisotropic universe models and application to the 5-yr WMAP data," Astrophys. J. 690, 1807 (2009) [arXiv:0807.2242 [astro-ph]].

[33] C. L. Bennett, R. S. Hill, G. Hinshaw, D. Larson, K. M. Smith, J. Dunkley, B. Gold and M. Halpern et al., "Seven-Year Wilkinson Microwave Anisotropy Probe (WMAP) Observations: Are There Cosmic Microwave Background Anomalies?," Astrophys. J. Suppl. 192, 17 (2011) [arXiv:1001.4758 [astro-ph.CO]].

[34] P. A. R. Ade et al. [Planck Collaboration], "Planck 2013 results. XXIII. Isotropy and statistics of the CMB," arXiv:1303.5083 [astro-ph.CO].

[35] S. 'i. Ando and M. Kamionkowski, "Nonlinear Evolution of Anisotropic Cosmological Power," Phys. Rev. Lett. 100, 071301 (2008) [arXiv:0711.0779 [astro-ph]].

[36] A. R. Pullen and C. M. Hirata, "Non-detection of a statistically anisotropic power spectrum in large-scale structure," JCAP 1005, 027 (2010) [arXiv:1003.0673 [astro-ph.CO]].

[37] D. Seery, M. S. Sloth and F. Vernizzi, "Inflationary trispectrum from graviton exchange," JCAP 0903, 018 (2009) [arXiv:0811.3934 [astro-ph]].

[38] D. Jeong and M. Kamionkowski, "Clustering Fossils from the Early Universe," Phys. Rev. Lett. 108, 251301 (2012) [arXiv:1203.0302 [astro-ph.CO]].

[39] D. Jeong and F. Schmidt, "Large-Scale Structure with Gravitational Waves I: Galaxy Clustering," Phys. Rev. D 86, 083512 (2012) [arXiv:1205.1512 [astro-ph.CO]].

[40] F. Schmidt, E. Pajer and M. Zaldarriaga, "Large-Scale Structure and Gravitational Waves III: 
Tidal Effects," Phys. Rev. D 89, 083507 (2014) [arXiv:1312.5616 [astro-ph.CO]].

[41] P. Catelan, M. Kamionkowski and R. D. Blandford, "Intrinsic and extrinsic galaxy alignment," Mon. Not. Roy. Astron. Soc. 320, L7 (2001) [astro-ph/0005470].

[42] J. M. Maldacena, "Non-Gaussian features of primordial fluctuations in single field inflationary models," JHEP 0305, 013 (2003) [astro-ph/0210603].

[43] V. Sreenath, R. Tibrewala and L. Sriramkumar, "Numerical evaluation of the three-point scalar-tensor cross-correlations and the tensor bi-spectrum," JCAP 1312, 037 (2013) [arXiv:1309.7169 [astro-ph.CO]].

[44] V. Sreenath and L. Sriramkumar, "Examining the consistency relations describing the three-point functions involving tensors," arXiv:1406.1609 [astro-ph.CO].

[45] P. Creminelli and M. Zaldarriaga, "Single field consistency relation for the 3-point function," JCAP 0410, 006 (2004) [astro-ph/0407059].

[46] A. Kehagias and A. Riotto, "Operator Product Expansion of Inflationary Correlators and Conformal Symmetry of de Sitter," Nucl. Phys. B 864, 492 (2012) [arXiv:1205.1523 [hep-th]].

[47] P. Creminelli, A. Joyce, J. Khoury and M. Simonovic, "Consistency Relations for the Conformal Mechanism," JCAP 1304, 020 (2013) [arXiv:1212.3329].

[48] W. D. Goldberger, L. Hui and A. Nicolis, "One-particle-irreducible consistency relations for cosmological perturbations," Phys. Rev. D 87, no. 10, 103520 (2013) [arXiv:1303.1193 [hep-th]].

[49] K. Hinterbichler, L. Hui and J. Khoury, "An Infinite Set of Ward Identities for Adiabatic Modes in Cosmology," JCAP 1401, 039 (2014) [arXiv:1304.5527 [hep-th]].

[50] A. Kehagias and A. Riotto, "Conformal Symmetries of FRW Accelerating Cosmologies," Nucl. Phys. B 884, 547 (2014) [arXiv:1309.3671 [hep-th]].

[51] S. Kundu, "Non-Gaussianity Consistency Relations, Initial States and Back-reaction," JCAP 1404, 016 (2014) [arXiv:1311.1575 [astro-ph.CO]].

[52] L. Berezhiani and J. Khoury, "Slavnov-Taylor Identities for Primordial Perturbations," JCAP 1402, 003 (2014) [arXiv:1309.4461 [hep-th]].

[53] L. Berezhiani, J. Khoury and J. Wang, "Non-Trivial Checks of Novel Consistency Relations," arXiv:1401.7991 [hep-th].

[54] L. Senatore and M. Zaldarriaga, "On Loops in Inflation II: IR Effects in Single Clock Inflation," JHEP 1301, 109 (2013) [JHEP 1301, 109 (2013)] [arXiv:1203.6354 [hep-th]].

[55] E. Pajer, F. Schmidt and M. Zaldarriaga, "The Observed Squeezed Limit of Cosmological Three-Point Functions," arXiv:1305.0824 [astro-ph.CO].

[56] M. H. Namjoo, H. Firouzjahi and M. Sasaki, "Violation of non-Gaussianity consistency relation in a single field inflationary model," Europhys. Lett. 101, 39001 (2013) [arXiv:1210.3692 [astro-ph.CO]].

[57] X. Chen, H. Firouzjahi, M. H. Namjoo and M. Sasaki, "A Single Field Inflation Model with Large Local Non-Gaussianity," Europhys. Lett. 102, 59001 (2013) [arXiv:1301.5699 [hep-th]].

X. Chen, H. Firouzjahi, E. Komatsu, M. H. Namjoo and M. Sasaki, "In-in and $\delta N$ calculations of the bispectrum from non-attractor single-field inflation," JCAP 1312, 039 (2013) [arXiv:1308.5341 [astro-ph.CO]].

[58] W. H. Kinney, "Horizon crossing and inflation with large eta," Phys. Rev. D 72, 023515 (2005) [gr-qc/0503017].

[59] S. Endlich, A. Nicolis and J. Wang, "Solid Inflation," JCAP 1310, 011 (2013) [arXiv:1210.0569 [hep-th]]. 
[60] S. Weinberg, "Adiabatic modes in cosmology," Phys. Rev. D 67, 123504 (2003) [astro-ph/0302326].

[61] C. Armendariz-Picon, T. Damour and V. F. Mukhanov, "k - inflation," Phys. Lett. B 458, 209 (1999) [hep-th/9904075].

[62] R. L. Arnowitt, S. Deser and C. W. Misner, "The Dynamics of general relativity," Gen. Rel. Grav. 40, 1997 (2008) [gr-qc/0405109].

[63] J. S. Schwinger, "Brownian motion of a quantum oscillator," J. Math. Phys. 2, 407 (1961).

[64] S. Weinberg, "Quantum contributions to cosmological correlations," Phys. Rev. D 72, 043514 (2005) [hep-th/0506236].

[65] S. Endlich and A. Nicolis, "The incompressible fluid revisited: vortex-sound interactions," arXiv:1303.3289 [hep-th].

[66] A. Nicolis, R. Penco and R. A. Rosen, "Relativistic Fluids, Superfluids, Solids and Supersolids from a Coset Construction," arXiv:1307.0517 [hep-th].

[67] S. Endlich, B. Horn, A. Nicolis and J. Wang, "The squeezed limit of the solid inflation three-point function," arXiv:1307.8114 [hep-th].

[68] N. Bartolo, S. Matarrese, M. Peloso and A. Ricciardone, "Anisotropy in solid inflation," JCAP 1308, 022 (2013) [arXiv:1306.4160 [astro-ph.CO]].

[69] M. Akhshik, R. Emami, H. Firouzjahi and Y. Wang, "Statistical Anisotropies in Gravitational Waves in Solid Inflation," arXiv:1405.4179 [astro-ph.CO].

[70] C. Cheung, P. Creminelli, A. L. Fitzpatrick, J. Kaplan and L. Senatore, "The Effective Field Theory of Inflation," JHEP 0803, 014 (2008) [arXiv:0709.0293 [hep-th]].

[71] A. R. Liddle, "Can the gravitational wave background from inflation be detected locally?," Phys. Rev. D 49, 3805 (1994) [Erratum-ibid. D 51, 4603 (1995)] [gr-qc/9307036].

[72] R. Bar-Kana, "Limits on direct detection of gravitational waves," Phys. Rev. D 50, 1157 (1994) [astro-ph/9401050].

[73] M. S. Turner, "Detectability of inflation produced gravitational waves," Phys. Rev. D 55, 435 (1997) [astro-ph/9607066].

[74] "Direct detection of the inflationary gravitational wave background," Phys. Rev. D 73, 023504 (2006) [astro-ph/0506422].

[75] T. L. Smith, M. Kamionkowski and A. Cooray, "The inflationary gravitational-wave background and measurements of the scalar spectral index," Phys. Rev. D 78, 083525 (2008) [arXiv:0802.1530 [astro-ph]].

[76] S. Chongchitnan and G. Efstathiou, "Prospects for direct detection of primordial gravitational waves," Phys. Rev. D 73, 083511 (2006) [astro-ph/0602594].

[77] S. Kuroyanagi, S. Tsujikawa, T. Chiba and N. Sugiyama, "Implications of the B-mode Polarization Measurement for Direct Detection of Inflationary Gravitational Waves," arXiv:1406.1369 [astro-ph.CO].

[78] R. Jinno, T. Moroi and T. Takahashi, "Studying Inflation with Future Space-Based Gravitational Wave Detectors," arXiv:1406.1666 [astro-ph.CO].

[79] D. Jeong, F. Schmidt and C. M. Hirata, "Large-scale clustering of galaxies in general relativity," Phys. Rev. D 85, 023504 (2012) [arXiv:1107.5427 [astro-ph.CO]].

[80] F. Arroja and K. Koyama, "Non-gaussianity from the trispectrum in general single field inflation," Phys. Rev. D 77, 083517 (2008) [arXiv:0802.1167 [hep-th]].

[81] N. Bartolo, E. Dimastrogiovanni and A. Vallinotto, "One-loop corrections to the power 
spectrum in general single-field inflation," JCAP 1011, 003 (2010) [arXiv:1006.0196 [astro-ph.CO]].

[82] P. R. Jarnhus and M. S. Sloth, "de Sitter limit of inflation and nonlinear perturbation theory," JCAP 0802, 013 (2008) [arXiv:0709.2708 [hep-th]].

[83] J. Garriga and V. F. Mukhanov, "Perturbations in k-inflation," Phys. Lett. B 458, 219 (1999) [hep-th/9904176].

[84] D. Seery and J. E. Lidsey, "Primordial non-Gaussianities in single field inflation," JCAP 0506, 003 (2005) [astro-ph/0503692].

[85] X. Chen, M. -x. Huang, S. Kachru and G. Shiu, "Observational signatures and non-Gaussianities of general single field inflation," JCAP 0701, 002 (2007) [hep-th/0605045].

[86] X. Chen, H. Firouzjahi, M. H. Namjoo and M. Sasaki, "A Single Field Inflation Model with Large Local Non-Gaussianity," Europhys. Lett. 102, 59001 (2013) [arXiv:1301.5699 [hep-th]].

[87] A. A. Abolhasani, S. Baghram, H. Firouzjahi and M. H. Namjoo, "Asymmetric Sky from the Long Mode Modulations," Phys. Rev. D 89, 063511 (2014) [arXiv:1306.6932 [astro-ph.CO]].

[88] P. Adshead and M. Wyman, "Gauge-flation trajectories in Chromo-Natural Inflation," Phys. Rev. D 86, 043530 (2012) [arXiv:1203.2264 [hep-th]].

[89] P. Adshead and M. Wyman, "Chromo-Natural Inflation: Natural inflation on a steep potential with classical non-Abelian gauge fields," Phys. Rev. Lett. 108, 261302 (2012) [arXiv:1202.2366 [hep-th]].

[90] M. M. Sheikh-Jabbari, "Gauge-flation Vs Chromo-Natural Inflation," Phys. Lett. B 717, 6 (2012) [arXiv:1203.2265 [hep-th]].

[91] E. Dimastrogiovanni, M. Fasiello and A. J. Tolley, "Low-Energy Effective Field Theory for Chromo-Natural Inflation," JCAP 1302, 046 (2013) [arXiv:1211.1396 [hep-th]].

[92] E. Dimastrogiovanni and M. Peloso, "Stability analysis of chromo-natural inflation and possible evasion of Lyth's bound," Phys. Rev. D 87, 103501 (2013) [arXiv:1212.5184 [astro-ph.CO]].

[93] A. Maleknejad, M. M. Sheikh-Jabbari and J. Soda, "Gauge Fields and Inflation," Phys. Rept. 528, 161 (2013) [arXiv:1212.2921 [hep-th]]. 